\title{
The Method of Fokas for Solving Linear Partial Differential Equations*
}

\author{
Bernard Deconinck ${ }^{\dagger}$ \\ Thomas Trogdon \\ Vishal Vasan ${ }^{\S}$
}

\begin{abstract}
The classical methods for solving initial-boundary-value problems for linear partial differential equations with constant coefficients rely on separation of variables and specific integral transforms. As such, they are limited to specific equations, with special boundary conditions. Here we review a method introduced by Fokas, which contains the classical methods as special cases. However, this method also allows for the equally explicit solution of problems for which no classical approach exists. In addition, it is possible to elucidate which boundary-value problems are well posed and which are not. We provide examples of problems posed on the positive half-line and on the finite interval. Some of these examples have solutions obtainable using classical methods, and others do not. For the former, it is illustrated how the classical methods may be recovered from the more general approach of Fokas.
\end{abstract}

Key words. partial differential equations, complex analysis, evolution equations

AMS subject classifications. $35-01,35 \mathrm{E} 05$

DOI. $10.1137 / 110821871$

I. Introduction. The canonical first course in partial differential equations (PDEs) focuses on explicit solution methods for problems for which such a solution can be found. The solution techniques presented include the method of separation of variables, Fourier series and transforms, Laplace and other integral transforms, Green's functions, etc. Standard textbooks for such courses include [4, 11, 12, 13, 17], each with their own amount of rigor. These textbooks focus almost all of their attention on scalar equations that are first- or second-order in any independent variable. In this context, an initial- or boundary-value problem (IVP or BVP) is considered solved if an explicit ${ }^{1}$ expression is constructed for the dependent variable as a function of the in-

\footnotetext{
* Received by the editors January 24, 2011; accepted for publication (in revised form) November 2, 2012; published electronically February 6, 2014.

http://www.siam.org/journals/sirev/56-1/82187.html

${ }^{\dagger}$ Department of Applied Mathematics, University of Washington, Seattle, WA 98195-2420 (bernard@amath.washington.edu).

${ }^{\ddagger}$ Courant Institute of Mathematical Sciences, New York University, New York, NY 10012 (tom. trogdon@gmail.com).

$\S^{\S}$ Department of Mathematics, Pennsylvania State University, University Park, PA 16802 (vasan@ math.psu.edu).

${ }^{1}$ For our purposes, an explicit expression is one whose evaluation is the topic of a lower-level course. For instance, the ordinary differential equation (ODE) $y^{\prime}+2 x y=1$ has an explicit solution since it is possible to express the solution in terms of operations whose evaluation is the topic of calculus, even though not all antiderivatives can be written in terms of elementary functions: $y=\exp \left(-x^{2}\right)\left(c+\int_{0}^{x} \exp \left(s^{2}\right) d s\right)$, with $c$ an arbitrary constant. Similarly, Fourier series, integrals in the complex plane, etc., give rise to explicit solutions of PDEs.
} 
dependent variables and of the given initial and boundary conditions. This expression is typically given as an infinite series or an integral, or a combination of both.

In this paper, we advocate a different method from those taught in any course. The method is relatively new. It was discovered by A. S. Fokas in his quest to generalize the method of inverse scattering, which solves the IVP for $x \in \mathbb{R}$ for socalled soliton equations, to BVPs posed either on the half-line $x \geq 0$ or on the finite interval $x \in[0, L][6,7,9]$. It was observed immediately $[8,10]$ that the method also produces interesting results for linear equations, which is our focus here. Just like the classical methods, the method of Fokas produces an explicit solution for the dependent variable $u(x, t)$. The solution formula is given in terms of one or more integrals along paths in the complex plane of an auxiliary variable $k$. All $x$ and $t$ dependence in the solution formula is entirely explicit. Although the method is more general, we restrict our attention to scalar problems with one spatial independent variable $x$ and one temporal independent variable $t$. We present the method of Fokas as a valuable addition to the standard methods taught, for several reasons.

- It encompasses the standard methods. For those cases where a standard method produces an explicit solution, Fokas's method does so as well. In fact, as we show in several examples below, the resulting solution formulas are equivalent, as they should be.

- It is more general than the standard methods: we are able to produce solution formulas for many problems where the classical methods cannot. This is particularly clear for problems containing higher than second-order derivatives.

- While the standard methods are a collection of situation-specific approaches, tailored to specific equations and boundary conditions, the method of Fokas produces a solution using the same ideas for all these different problems, with any differences appearing only in the calculational details.

- In addition to producing an explicit formula for the solution, the method allows one to determine in a straightforward way how many and which boundary conditions result in a well-posed problem. Especially for BVPs for equations with higher than second-order derivatives, this is a nontrivial issue.

- Efficient evaluation of the solution can be obtained from the explicit solution formula using different means: parametrization of the contours resulting in integrals that are easily evaluated numerically, asymptotic methods such as steepest descent, the residue theorem, and so on.

- The background required for the method is limited to knowledge of the Fourier and inverse Fourier transform pair, the residue theorem, and Jordan's lemma; see [1] or [3], for instance. In other words, even if no course on complex analysis precedes the first course on PDEs, the necessary material is easily introduced in three or four lectures.

Our introduction to the method of Fokas proceeds mostly by example. More details and general arguments can be found in [9]. We start by revisiting the IVP on the whole line in the framework of the new method; see section 2 . This merely results in the construction of the familiar Fourier transform expression for the solution, but it does allow us to generalize more naturally the approach to the half-line and finite interval problems more naturally. This is done in sections 3 and 4 , respectively, which comprise the bulk of the paper. The reader will find examples of problems that can be solved using the standard methods, in which cases we demonstrate the equivalence of the classical results with those obtained using Fokas's method. Other examples illustrate the use of the method in situations where the standard methods fail or are unable to proceed. Our emphasis is not on rigor and no comments are made about 
function spaces. Rather, in the vein of a first course on PDEs (see, for instance, $[4,12])$, we assume whatever conditions are necessary for the calculations to proceed: for instance, functions are at least as differentiable as dictated by the equation and boundary and initial conditions are compatible at $(x, t)=(0,0)$ and, for the finite interval, at $(x, t)=(L, 0)$.

2. The Initial-Value Problem on the Whole Line. In contrast to a traditional first course on PDEs, we begin by considering the IVP for linear constant coefficient equations on the entire real line. In order to start with a concrete example, consider the IVP for the heat equation:

$$
\begin{aligned}
& q_{t}=q_{x x}, \quad x \in \mathbb{R}, t \in(0, T], \\
& q(x, 0)=q_{0}(x), \quad x \in \mathbb{R},
\end{aligned}
$$

where subscripts denote partial differentiation. Here $T$ is a positive real number, and we impose the boundary condition that $q(x, t) \rightarrow 0$ sufficiently fast as $|x| \rightarrow \infty$ for all $t \geq 0$. The solution to this problem is given by

$$
q(x, t)=\frac{1}{2 \pi} \int_{-\infty}^{\infty} \hat{q}_{0}(k) e^{i k x-\omega(k) t} d k,
$$

where $\omega(k)=k^{2}$ is the dispersion relation ${ }^{2}$ for the heat equation and $\hat{q}_{0}(k)$ is the Fourier transform of the initial condition:

$$
\hat{q}_{0}(k)=\int_{-\infty}^{\infty} q_{0}(x) e^{-i k x} d x .
$$

Further, letting $T \rightarrow \infty$ does not affect the validity of the solution. In a traditional text, this result is obtained by taking the Fourier transform of the original PDE (2.1a). This results in a one-parameter family of ODEs with parameter $k$. Solving this ODE gives the Fourier transform of the solution at any time $t$. Using the inverse transform, the above result is obtained.

In the new approach, we start by rewriting the heat equation as a one-parameter family of PDEs in divergence form,

$$
\partial_{t} \rho(x, t, k)+\partial_{x} j(x, t, k)=0,
$$

where

$$
\rho(x, t, k)=e^{-i k x+\omega(k) t} q,
$$

with $k \in \mathbb{C}$. We refer to (2.4) as the local relation. The explicit form of $j$ is easily obtained using the reverse product rule:

$$
\begin{aligned}
\left(e^{-i k x+\omega(k) t} q\right)_{t}= & \omega(k) e^{-i k x+\omega(k) t} q+e^{-i k x+\omega(k) t} q_{t} \\
= & \omega(k) e^{-i k x+\omega(k) t} q+e^{-i k x+\omega(k) t} q_{x x} \\
= & \omega(k) e^{-i k x+\omega(k) t} q+\left(e^{-i k x+\omega(k) t} q_{x}\right)_{x}+i k e^{-i k x+\omega(k) t} q_{x} \\
= & \omega(k) e^{-i k x+\omega(k) t} q+\left(e^{-i k x+\omega(k) t} q_{x}\right)_{x}+\left(i k e^{-i k x+\omega(k) t} q\right)_{x} \\
& -k^{2} e^{-i k x+\omega(k) t} q \\
= & \left(e^{-i k x+\omega(k) t}\left(q_{x}+i k q\right)\right)_{x}
\end{aligned}
$$

so that $j=-e^{-i k x+\omega(k) t}\left(q_{x}+i k q\right)$. Note that this calculation also determines $\omega(k)$.

\footnotetext{
${ }^{2}$ For consistency with the literature on Fokas's method [9], we adopt the convention that the dispersion relation $\omega(k)$ is found by substitution of $\exp (i k x-\omega(k) t)$ in the PDE.
} 


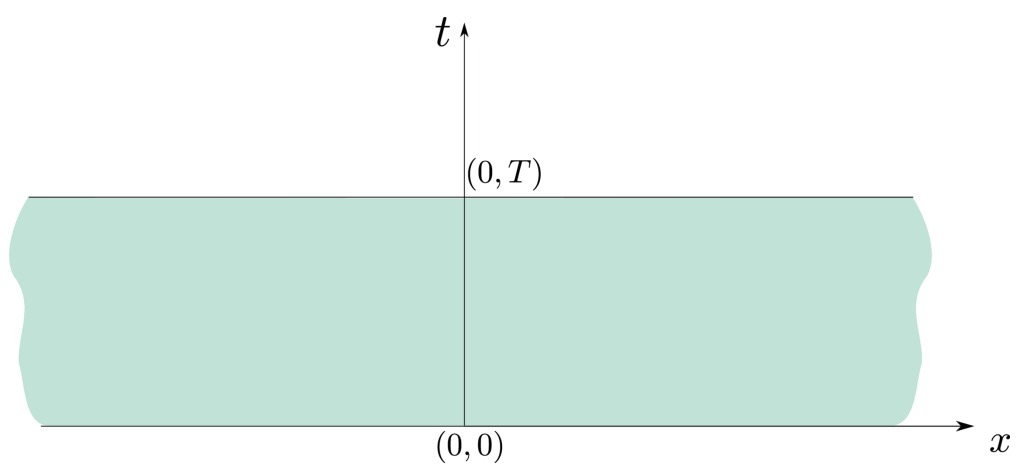

Fig. 2.I The region of integration in the $(x, t)$-plane for problems posed on the whole-line.

We are now in a position to apply Green's theorem in the $(x, t)$-plane. Consider (2.5) on an infinite horizontal strip $\mathcal{D}$ of height $T$ (see Figure 2.1). In what follows, $s$ is a temporal dummy variable. Using the decay properties of the solution where necessary, we have

$$
\begin{gathered}
\iint_{\mathcal{D}}\left(\left[e^{-i k x+\omega(k) s} q(x, s)\right]_{s}-\left[e^{-i k x+\omega(k) s}\left(q_{x}(x, s)+i k q(x, s)\right)\right]_{x}\right) d s d x=0 \\
\begin{array}{l}
\text { Green's theorem) } \\
\Rightarrow
\end{array} \int_{\partial \mathcal{D}}\left(e^{-i k x+\omega(k) s} q(x, s) d x+e^{-i k x+\omega(k) s}\left(q_{x}(x, s)+i k q(x, s)\right) d s\right)=0 \\
\Rightarrow \quad \int_{-\infty}^{\infty} e^{-i k x} q_{0}(x) d x-\int_{-\infty}^{\infty} e^{-i k x+\omega(k) T} q(x, T) d x=0 \\
\quad \Rightarrow \quad \int_{-\infty}^{\infty} e^{-i k x} q(x, T) d x=e^{-\omega(k) T} \int_{-\infty}^{\infty} e^{-i k x} q_{0}(x) d x .
\end{gathered}
$$

The contribution of the second integrand on the second line vanishes due to the decay properties of the solution as $|x| \rightarrow \infty$. In later sections, such terms bring in the contributions from the boundary data. The last line relates the Fourier transform of the solution at time $T$ to the Fourier transform of the initial value $q_{0}(x)$, as expected. Inverting the transform we obtain the solution (2.2).

Let us take a step back to review our work. It appears we have obtained the traditional result in a roundabout way. However, progress has been made. The procedure used here is suitable for solving problems other than those posed on the whole-line. By working with different domains (such as those used below) for the application of Green's theorem to the local relation, we obtain the integral expressions for solutions to the corresponding BVP.

Remarks.

- The procedure above works equally well for equations other than the heat equation. In fact, the solution formula (2.2) stands as is, as long as the appropriate dispersion relation is filled in. We do not elaborate on this here; details are found, for instance, in the appendix of [2]. For BVPs, more detail is found below.

- The conversion of the PDE to the local relation (2.4) is always possible for linear constant coefficient PDEs [9]. The explicit form of $j(x, t, k)$ in terms of $\omega(k)$, avoiding the reverse product rule, is given in (3.35). See section 3.5 for more detail. 


\section{The Problem on the Half-Line.}

3.I. The Heat Equation with Dirichlet Boundary Conditions. In section 2 the heat equation on the whole real line was solved. The success of this method was due to the properties of the Fourier transform, which incorporates the "boundary condition" of decay at infinity. In this section we solve the heat equation on the half-line with Dirichlet boundary data:

$$
\begin{aligned}
q_{t} & =q_{x x}, & x>0, t \in(0, T], \\
q(x, 0) & =q_{0}(x), & x \geq 0, \\
q(0, t) & =g_{0}(t), & t \in[0, T] .
\end{aligned}
$$

This is a standard textbook problem, easily solved using classical techniques. As mentioned, its inclusion here serves the purpose of allowing the reader to see how problems solvable using the classical methods can be solved using the method of Fokas. We begin by considering the local relation of the heat equation (2.5), which holds independent of the solution domain and the boundary conditions as it is a local statement. Applying Green's theorem to this equation with the domain of integration $\mathcal{R}=\{x \geq 0,0<t \leq T\}$ (see Figure 3.1) we obtain

$$
\begin{aligned}
& \int_{\partial \mathcal{R}}\left(e^{-i k x+\omega(k) s} q(x, s) d x+e^{-i k x+\omega(k) s}\left(q_{x}(x, s)+i k q(x, s)\right) d s\right)=0 \\
\Rightarrow & \int_{0}^{\infty} e^{-i k x} q_{0}(x) d x-\int_{0}^{\infty} e^{-i k x+\omega(k) T} q(x, T) d x \\
& -\int_{0}^{T} e^{\omega(k) s}\left(q_{x}(0, s)+i k q(0, s)\right) d s=0 \\
\Rightarrow & \int_{0}^{\infty} e^{-i k x} q_{0}(x) d x-\int_{0}^{T} e^{\omega(k) s}\left(q_{x}(0, s)+i k q(0, s)\right) d s \\
& =e^{\omega(k) T} \int_{0}^{\infty} e^{-i k x} q(x, T) d x \\
(3.2) \Rightarrow & \hat{q}_{0}(k)-\left[\tilde{g}_{1}(\omega(k), T)+i k \tilde{g}_{0}(\omega(k), T)\right]=e^{\omega(k) T} \hat{q}(k, T),
\end{aligned}
$$

where $\partial \mathcal{R}$ denotes the boundary of the domain $\mathcal{R}$, oriented so that the domain $\mathcal{R}$ is on the left when the boundary is traversed. Further, $\hat{q}_{0}, \hat{q}$ are the Fourier transforms

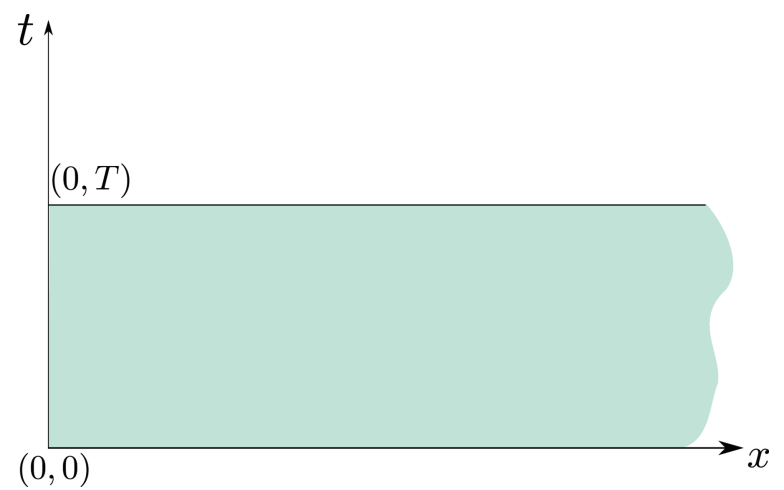

Fig. 3.I The region of integration in the $(x, t)$-plane for BVPs posed on the positive half-line. 
of the initial condition and the solution at time $T$, respectively. Similarly, $\tilde{g}_{0}$ and $\tilde{g}_{1}$ are defined in terms of the boundary data as

$$
\tilde{g}_{0}(\omega, T)=\int_{0}^{T} e^{\omega s} q(0, s) d s, \quad \tilde{g}_{1}(\omega, T)=\int_{0}^{T} e^{\omega s} q_{x}(0, s) d s .
$$

We refer to (3.2) as the global relation for the heat equation on the half-line. The time transforms $\tilde{g}_{0}, \tilde{g}_{1}$ arise because of the presence of the boundary at $x=0$. For the problem under consideration, the Dirichlet data are given; thus $\tilde{g_{0}}$ is determined, whereas $\tilde{g_{1}}$ is not.

The spectral parameter $k$ associated with the Fourier transform is typically real. However, the terms in the global relation (3.2) are analytic for $\operatorname{Im}(k)<0$. Indeed, the Fourier transforms may be analytically continued into the lower half of the complex $k$ plane due to the exponential decay there, assuming sufficient decay of $q(x, t)$ for large $x$ and all $t$. Further, the time transforms $\left(\tilde{g}_{0}, \tilde{g}_{1}\right)$ are entire functions (analytic and bounded for all finite $k$ ).

Remark. The continuation of the global relation into the lower half-plane, for the equation on the half-line, should be contrasted with the "global relation" for the whole-line case (2.6), where such an extension to complex $k$ is not possible.

The global relation (3.2) is equally valid for any $t \in(0, T]$. Replacing $T$ by $t$ in the global relation (3.2) and inverting the Fourier transform, we arrive at an integral expression for $q(x, t)$ :

$$
\begin{aligned}
\hat{q}_{0}(k) & -\left[\tilde{g}_{1}(\omega(k), t)+i k \tilde{g}_{0}(\omega(k), t)\right]=e^{\omega(k) t} \hat{q}(k, t) \\
\Rightarrow \quad q(x, t) & =\frac{1}{2 \pi} \int_{-\infty}^{\infty} e^{i k x-\omega(k) t} \hat{q}_{0}(k) d k \\
- & \frac{1}{2 \pi} \int_{-\infty}^{\infty} e^{i k x-\omega(k) t}\left[\tilde{g}_{1}(\omega(k), t)+i k \tilde{g}_{0}(\omega(k), t)\right] d k .
\end{aligned}
$$

Let $D=\{k \in \mathbb{C}: \operatorname{Re}(\omega(k))<0\}$. Further, let $D^{+}=D \cap \mathbb{C}^{+}$, where $\mathbb{C}^{+}=\{k \in$ $\mathbb{C}: \operatorname{Im}(k)>0\}$. The integrand of the second integral in (3.3) is entire and decays as $k \rightarrow \infty$ for $k \in \mathbb{C}^{+} \backslash D^{+}$. Consider a contour $\mathcal{C}=[-R, R] \cup \mathcal{C}_{R_{2}} \cup \mathcal{C}_{\partial D^{+}} \cup \mathcal{C}_{R_{1}}$ as shown in bold in Figure 3.2. Let $\mathcal{C}_{\partial D^{+}}$be the part of $\mathcal{C}$ on the boundary of $D^{+}$and $\mathcal{C}_{R_{1}}$, and let $\mathcal{C}_{R_{2}}$ be circular arcs of radius $R$. Using the analyticity of the integrand,

$$
\int_{\mathcal{C}} e^{i k x-\omega(k) t} \tilde{g}(\omega(k), t) d k=\left(\int_{-R}^{R}+\int_{\mathcal{C}_{R_{2}}}+\int_{\mathcal{C}_{\partial D^{+}}}+\int_{\mathcal{C}_{R_{1}}}\right) e^{i k x-\omega(k) t} \tilde{g}(\omega(k), t) d k=0,
$$

where $\tilde{g}(\omega(k), t)=\tilde{g}_{1}(\omega(k), t)+i k \tilde{g}_{0}(\omega(k), t)$. Taking the limit $R \rightarrow \infty$ of the above expression, we observe that the contour $\mathcal{C}_{\partial D^{+}}$becomes the contour $-\partial D^{+}$. The negative sign arises from the convention that the positive orientation of a boundary is defined so that the region is to the left as the boundary is traversed. Further, an application of Jordan's lemma in the wedge-like regions shows that for large $R$, the contribution of the integrals along $\mathcal{C}_{R_{1}}$ and $\mathcal{C}_{R_{2}}$ vanishes. We obtain that the integral of $\exp (i k x-\omega(k) t) \tilde{g}(\omega(k), t)$ along the real line may be replaced by one along $\partial D^{+}$. Thus

$q(x, t)=\frac{1}{2 \pi} \int_{-\infty}^{\infty} e^{i k x-\omega(k) t} \hat{q}_{0}(k) d k-\frac{1}{2 \pi} \int_{\partial D^{+}} e^{i k x-\omega(k) t}\left[\tilde{g}_{1}(\omega(k), t)+i k \tilde{g}_{0}(\omega(k), t)\right] d k$. 


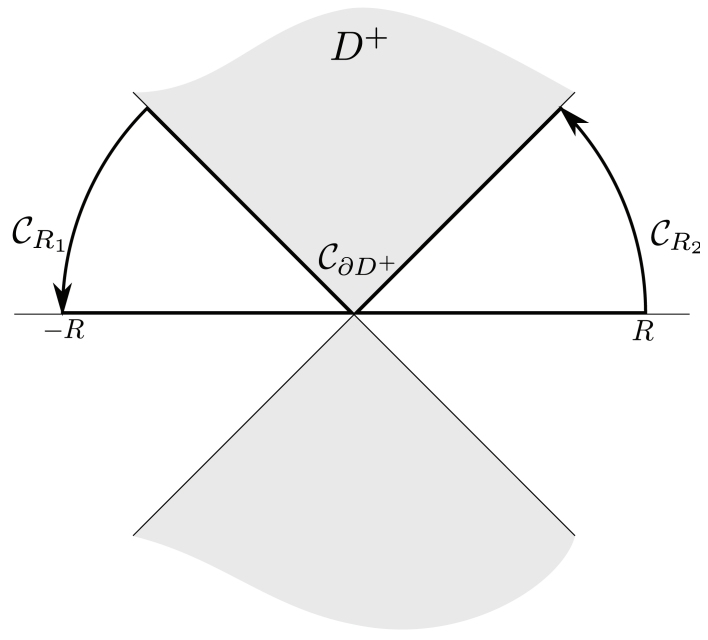

Fig. 3.2 The domain $D$ for the heat equation is indicated in gray. The contour $\mathcal{C}$, shown in bold, is used to justify the deformation of the line integral along the real line up to the boundary of $D^{+}$.

Summarizing the argument, the contour $\mathcal{C}$ may be replaced by $\partial\left(\mathbb{C}^{+} \backslash D^{+}\right)$as $R \rightarrow$ $\infty$. The integral of $\exp (i k x-\omega(k) t) \tilde{g}(\omega(k), t)$ along $\partial\left(\mathbb{C}^{+} \backslash D^{+}\right)$is zero due to the analyticity and decay properties of the integrand. Adding this integral to the righthand side of (3.3), we obtain (3.5).

Although (3.5) is an expression for $q(x, t)$, it does not present a solution since it depends on boundary data we have not prescribed through $\tilde{g}_{1}$, the transform of the Neumann data. To resolve this problem, we could solve the global relation (3.2) for $\tilde{g}_{1}$. This results in an expression for $\tilde{g}_{1}$ valid in $\mathbb{C}^{-}=\{k: \operatorname{Im}(k)<0\}$, whereas (3.5) requires an expression for $\tilde{g}_{1}$ valid along $\partial D^{+}$. To this end we seek a transform that maps the contour $\partial D^{+}$to a contour in the lower half-plane $\mathbb{C}^{-}$but leaves $\tilde{g}_{1}(\omega(k), t)$ invariant. Thus we turn to the discrete symmetries of $\omega(k)=k^{2}$. The dispersion relation $\omega(k)$ is invariant under the transform $k \rightarrow-k$. Applying this transformation to the global relation (3.2) (and replacing $T$ by $t$ ), we have

$$
\hat{q}(-k, t)=e^{-\omega(k) t} \hat{q}_{0}(-k)-e^{-\omega(k) t}\left[\tilde{g}_{1}(\omega(k), t)-i k \tilde{g}_{0}(\omega(k), t)\right], \quad \operatorname{Im}(k) \geq 0 .
$$

Solving this version of the global relation yields an expression for $\tilde{g}_{1}(\omega(k), t)$ which is valid along $\partial D^{+}$. The integral expression for $q(x, t)$ becomes

$$
\begin{aligned}
q(x, t)= & \frac{1}{2 \pi} \int_{-\infty}^{\infty} e^{i k x-\omega(k) t} \hat{q}_{0}(k) d k-\frac{1}{2 \pi} \int_{\partial D^{+}} e^{i k x-\omega(k) t}\left[2 i k \tilde{g}_{0}(\omega(k), t)+\hat{q}_{0}(-k)\right] d k \\
& +\frac{1}{2 \pi} \int_{\partial D^{+}} e^{i k x} \hat{q}(-k, t) d k .
\end{aligned}
$$

The above expression does not depend on the unknown boundary data. However, the function we wish to solve for, $q(x, t)$, also appears in the third integral on the right-hand side. Using analyticity, this problem is resolved as follows. The function $\hat{q}(-k, t)$ is bounded and analytic in $\mathbb{C}^{+}$with $\hat{q}(-k, t) \rightarrow 0$ uniformly as $k \rightarrow \infty$. This implies that the integral of $\exp (i k x) q(-k, t)$ along a closed, bounded curve in $\mathbb{C}^{+}$ 


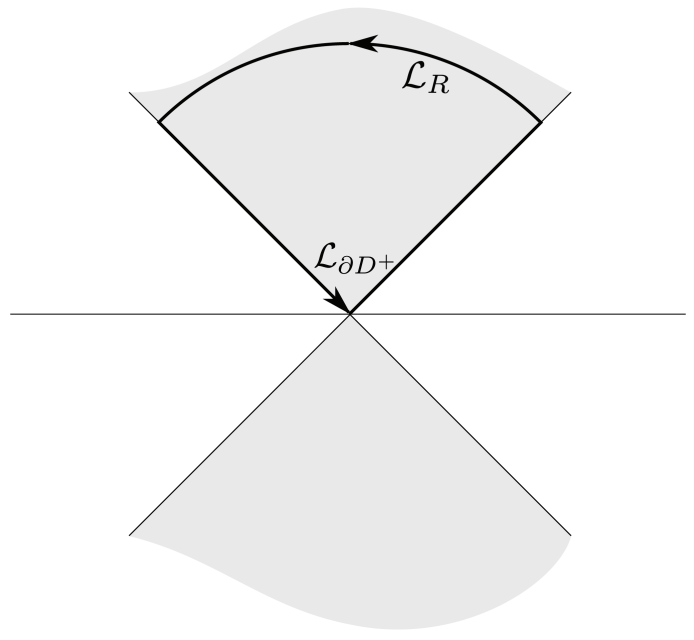

Fig. 3.3 The contour $\mathcal{L}$ is shown in bold. Application of Cauchy's integral theorem using this contour allows one to eliminate the contribution of $\hat{q}(-k, t)$ from the integral expression (3.7).

vanishes. In particular, we consider a closed curve $\mathcal{L}=\mathcal{L}_{\partial D^{+}} \cup \mathcal{L}_{R}$, where $\mathcal{L}_{\partial D^{+}}=$ $\partial D^{+} \cap\{k:|k|<R\}$ and $\mathcal{L}_{R}=\left\{k \in D^{+}:|k|=R\right\}$ (see Figure 3.3). We have

$$
\int_{\mathcal{L}} e^{i k x} \hat{q}(-k, t) d k=\int_{\mathcal{L}_{\partial D^{+}}} e^{i k x} \hat{q}(-k, t) d k+\int_{\mathcal{L}_{R}} e^{i k x} \hat{q}(-k, t) d k=0 .
$$

If it can be shown that the integral along $\mathcal{L}_{R}$ vanishes for large $R$, then the third integral on the right-hand side of (3.7) must also vanish since the contour $\mathcal{L}_{\partial D^{+}}$ becomes $\partial D^{+}$as $R \rightarrow \infty$. From Jordan's lemma, the uniform decay of $\hat{q}(-k, t)$ for large $k$ is precisely the condition required for the integral to vanish. Thus

$$
q(x, t)=\frac{1}{2 \pi} \int_{-\infty}^{\infty} e^{i k x-\omega(k) t} \hat{q}_{0}(k) d k-\frac{1}{2 \pi} \int_{\partial D^{+}} e^{i k x-\omega(k) t}\left[\hat{q}_{0}(-k)+2 i k \tilde{g}_{0}(\omega(k), t)\right] d k
$$

is the solution to the Dirichlet problem for the heat equation on the half-line. The contours in the complex plane may be parameterized as desired.

Let us summarize the steps involved in this method.

1. Using the dispersion relation $\omega(k)$ we define the regions $D=\{k: \operatorname{Re}(\omega(k))<$ $0\}, D^{+}=D \cap \mathbb{C}^{+}$, and $D^{-}=D \cap \mathbb{C}^{-}$.

2. The PDE is rewritten as a one-parameter family of equations in divergence form. Applying Green's theorem in the $(x, t)$-plane, we obtain the global relation. By considering complex values for the spectral parameter $k$, we extend the domain of definition of the global relation. For problems posed on the half-line, the global relation is valid in $\operatorname{Im}(k) \leq 0$.

3. The global relation is solved for $\hat{q}(k, t)$. The integral expression for $q(x, t)$ is constructed by inverting the Fourier transform. The integral involving the boundary terms is deformed off the real line. For problems on the half-line, we deform up to $\partial D^{+}$.

4. The discrete symmetries $\nu(k)$ of $\omega(k), \omega(k)=\omega(\nu(k))$, are used to obtain additional versions of the global relation valid for $k$ in certain regions of $\mathbb{C}^{+}$. 
These additional global relations are solved simultaneously for the transforms of the unknown boundary data. The expressions thus obtained are substituted into the integral expression for $q(x, t)$.

5. The integral expression for $q(x, t)$ now depends on $\hat{q}(\nu(k), t)$. Analyticity considerations are used to evaluate the contribution of $\hat{q}(\nu(k), t)$. Typically this contribution vanishes. The functions $\hat{q}(\nu(k), t)$ and $\exp (i k x)$ are bounded and analytic in the upper half-plane. If the coefficient of $\exp (i k x) \hat{q}(\nu(k), t)$ in the integral expression is also analytic in $D^{+}$, then the contribution of this term is zero. For instance, this happens for the Dirichlet problem for the heat equation posed on the half-line where this coefficient is a constant. Let us now consider the case when this coefficient has a simple pole at some point $k=k_{0}$ in $D^{+}$. An application of the residue theorem shows that we need the value of $\hat{q}\left(\nu\left(k_{0}\right), t\right)$. At this point, the global relation is used once more. The global relation connects the transform of the solution at time $t$ to the transforms of both the known initial-boundary conditions and the unknown boundary conditions. Evaluating the global relation at $k=\nu\left(k_{0}\right)$, we obtain an expression for $\hat{q}\left(\nu\left(k_{0}\right), t\right)$. If this expression depends only on known initial-boundary conditions (i.e., the coefficients of the unknown terms add up to zero), we have solved the problem. The presence of unknown boundary conditions hints at an ill-posed problem. Note that we select only those transformations $\nu(k)$ whose image lies in the lower half-plane. Consequently, $\hat{q}\left(\nu\left(k_{0}\right), t\right)$ is defined.

Remarks.

- For the Neumann problem, when $q_{x}(0, t)$ is supplied as the boundary condition, we can just as easily solve the global relation for $\tilde{g}_{0}$. The integral involving $\hat{q}(-k, t)$ vanishes for the same reason as for the Dirichlet problem [9].

- The classical solution in terms of the sine transform can be recovered from the solution to the heat equation (3.9). Note that $\hat{q}_{0}(-k)$ is analytic and bounded in the upper half-plane and $\exp (-\omega(k) t) k \tilde{g}_{0}(\omega(k), t)$ is bounded and analytic in the region between $D^{+}$and the real line. Thus the contour $\partial D^{+}$may be deformed back to the real line:

$$
\begin{aligned}
q(x, t)= & \frac{1}{2 \pi} \int_{-\infty}^{\infty} e^{i k x-\omega(k) t} \hat{q}_{0}(k) d k \\
& -\frac{1}{2 \pi} \int_{-\infty}^{\infty} e^{i k x-\omega(k) t}\left(\hat{q}_{0}(-k)+2 i k \tilde{g}_{0}(\omega(k), t)\right) d k .
\end{aligned}
$$

By splitting the integral along the real line into integrals from $-\infty$ to 0 and from 0 to $\infty$, and using the definition of sine in terms of exponentials, we obtain

$$
q(x, t)=\frac{2}{\pi} \int_{0}^{\infty} e^{-\omega(k) t} \sin (k x)\left[\int_{0}^{\infty} \sin (k y) q(y, 0) d y-k \tilde{g}_{0}(\omega(k), t)\right] d k .
$$

This deformation back to the real line is not possible for all PDEs. In fact, the deformation is possible only when a classical transform exists. Also note that, unlike the integral representation (3.9), the sine transform solution is not uniformly convergent at $x=0$. Further, using methods like steepest descent or stationary phase, the contribution due to the boundary can be 
evaluated much more efficiently in (3.9) than in the classical sine transform solution; see [5].

- An alternative to the new method is the use of Laplace transforms in $t$. This results in multivalued integral kernels due to the presence of radicals. Consequently, the inversion of the Laplace transform involves contour integrals along branch cuts; see, for example, the example on page 358 of [17]. For spatial derivatives of order greater than two, this procedure quickly becomes far more involved. Further, the Laplace transform involves an integration over all time $t \geq 0$. This seems to contradict causality for evolution problems. For evolution equations we do not expect the solution at time $t=T$ to depend on times greater than $T$.

3.2. A Third-Order PDE with Dirichlet Boundary Conditions. As a second example consider the following problem, posed again on the half-line:

$$
\begin{aligned}
q_{t}+q_{x x x} & =0, & x \geq 0, t \in(0, T], \\
q(x, 0) & =q_{0}(x), & x \geq 0, \\
q(0, t) & =g_{0}(t), & t \in(0, T] .
\end{aligned}
$$

As before, we assume that $q(x, t)$ decays sufficiently fast as $x \rightarrow \infty$ for all $t \geq 0$. We follow the steps outlined in the previous section.

1. Dispersion relation. The dispersion relation for the $\mathrm{PDE}$ is $\omega(k)=-i k^{3}$. Using

$$
\omega(k)=-i|k|^{3}[\cos (3 \arg k)+i \sin (3 \arg k)],
$$

we define the region $D($ where $\operatorname{Re}(\omega(k))<0)$ as

$$
D=\left\{k: \arg k \in\left(\frac{\pi}{3}, \frac{2 \pi}{3}\right) \cup\left(\pi, \frac{4 \pi}{3}\right) \cup\left(\frac{5 \pi}{3}, 2 \pi\right)\right\},
$$

so that

$$
D^{+}=\left\{k: \arg k \in\left(\frac{\pi}{3}, \frac{2 \pi}{3}\right)\right\}
$$

and

$$
D^{-}=D_{1}^{-} \cup D_{2}^{-},
$$

where

$$
D_{1}^{-}=\left\{k: \arg k \in\left(\pi, \frac{4 \pi}{3}\right)\right\}, \quad D_{2}^{-}=\left\{k: \arg k \in\left(\frac{5 \pi}{3}, 2 \pi\right)\right\} .
$$

See Figure 3.4 for a depiction of these regions.

2. Global relation. Using the same method as before, we find the local relation

$$
\left(e^{-i k x+\omega(k) t} q\right)_{t}+\left(e^{-i k x+\omega(k) t}\left(q_{x x}+i k q_{x}+(i k)^{2} q\right)\right)_{x}=0
$$

which is easily verified. Integrating the above equation over the domain 


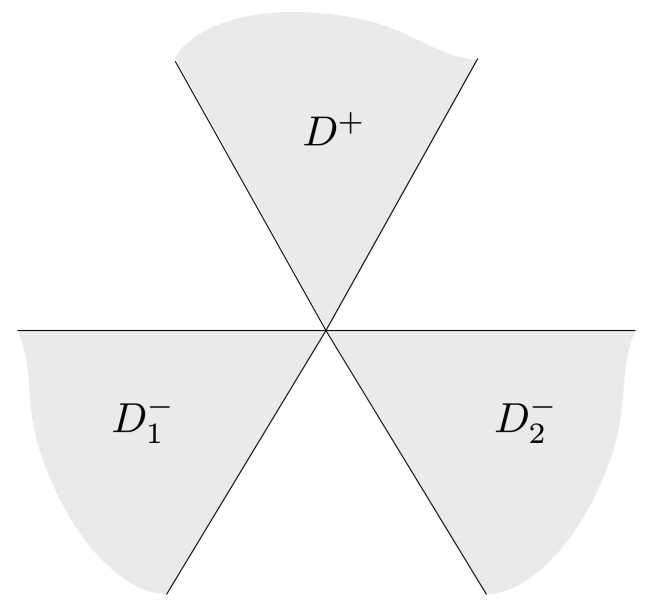

Fig. 3.4 Domain $D=\{k: \operatorname{Re}(\omega(k))<0\}$ for the third-order PDE (3.10a).

$\mathcal{R}=\{0 \leq x<\infty, 0<t \leq T\}$ and applying Green's theorem, we obtain

$$
\begin{aligned}
& \iint_{\mathcal{R}}\left(\left[e^{-i k x+\omega(k) s} q(x, s)\right]_{s}-\left[e^{-i k x+\omega(k) t}\left(-q_{x x}-i k q_{x}-(i k)^{2} q\right)\right]_{x}\right) d s d x=0 \\
\Rightarrow & \int_{\partial \mathcal{R}}\left(e^{-i k x+\omega(k) s} q(x, s) d x+e^{-i k x+\omega(k) s}\left(-q_{x x}(x, s)\right.\right. \\
& \left.\left.-i k q_{x}(x, s)-(i k)^{2} q(x, s)\right) d t\right)=0 \\
\Rightarrow & \int_{0}^{\infty} e^{-i k x} q(x, 0) d x-\int_{0}^{T} e^{\omega(k) s}\left(-q_{x x}(0, s)-i k q_{x}(0, s)-(i k)^{2} q(0, s)\right) d s \\
\Rightarrow & \quad \int_{0}^{\infty}(k)-\left[k^{2} \tilde{g}_{0}(\omega(k), T)-i k \tilde{g}_{1}(\omega(k), T)-\tilde{g}_{2}(\omega(k), T)\right]=e^{\omega(k) T} \hat{q}(k, T),
\end{aligned}
$$

where, as before, $\hat{q}_{0}(k)$ and $\hat{q}(k, T)$ represent the Fourier transform of the solution at time $t=0$ and time $t=T$. The time transforms of the boundary data are given by

$$
\tilde{g}_{i}(\omega, T)=\int_{0}^{T} e^{\omega s} \partial_{x}^{i} q(0, s) d s, i=0,1,2
$$

Equation (3.11) is the global relation for the third-order PDE (3.10a) posed on the positive half-line. Note that it is valid for $\operatorname{Im}(k) \leq 0$.

3. Integral expression. Let

$$
\tilde{g}(k, t)=k^{2} \tilde{g}_{0}(\omega(k), t)-i k \tilde{g}_{1}(\omega(k), t)-\tilde{g}_{2}(\omega(k), t)
$$

Replacing $T$ by $t$ in the global relation (3.11) and applying the inverse Fourier 
transform, we obtain

$$
\begin{aligned}
& e^{\omega(k) t} \hat{q}(k, t)=\hat{q}_{0}(k)-\tilde{g}(k, t) \\
\Rightarrow & \hat{q}(k, t)=e^{-\omega(k) t} \hat{q}_{0}(k)-e^{-\omega(k) t} \tilde{g}(k, t) \\
\Rightarrow & q(x, t)=\frac{1}{2 \pi} \int_{-\infty}^{\infty} e^{i k x-\omega(k) t} \hat{q}_{0}(k) d k-\frac{1}{2 \pi} \int_{-\infty}^{\infty} e^{i k x-\omega(k) t} \tilde{g}(k, t) d k \\
\Rightarrow & q(x, t)=\frac{1}{2 \pi} \int_{-\infty}^{\infty} e^{i k x-\omega(k) t} \hat{q}_{0}(k) d k-\frac{1}{2 \pi} \int_{\partial D^{+}} e^{i k x-\omega(k) t} \tilde{g}(k, t) d k,
\end{aligned}
$$

where the second integral on the last line has been deformed into the upper half complex plane up to the boundary of $D^{+}$.

4. Solving for the unknown boundary data. As for the heat equation, the integral expression for $q(x, t)$ does not represent a solution due to the presence of unknown boundary terms, here $\tilde{g}_{1}, \tilde{g}_{2}$. As before we use the discrete symmetries of $\omega(k)$. In this case, these are $\nu_{1}(k)=e^{\frac{2 \pi i}{3}} k$ and $\nu_{2}(k)=e^{\frac{4 \pi i}{3}} k$.

We observe that for $k \in D^{+}, \nu_{1}(k) \in D_{1}^{-}$and $\nu_{2}(k) \in D_{2}^{-}$. Applying these transformations to the global relation, for $k \in D^{+}$with $\alpha=e^{\frac{2 \pi i}{3}}$ we find

$$
\begin{aligned}
\hat{q}_{0}(\alpha k)-\alpha^{2} k^{2} \tilde{g}_{0}(\omega(k), t)+i \alpha k \tilde{g}_{1}(\omega(k), t)+\tilde{g}_{2}(\omega(k), t) & =e^{\omega(k) t} \hat{q}(\alpha k, t), \\
\hat{q}_{0}\left(\alpha^{2} k\right)-\alpha k^{2} \tilde{g}_{0}(\omega(k), t)+i \alpha^{2} k \tilde{g}_{1}(\omega(k), t)+\tilde{g}_{2}(\omega(k), t) & =e^{\omega(k) t} \hat{q}\left(\alpha^{2} k, t\right) .
\end{aligned}
$$

Given $q(x, 0)$ and $q(0, t)$ (or $\hat{q}_{0}(k)$ and $\left.\tilde{g}_{0}(\omega, t)\right)$ we may solve the above two equations for the two unknowns $\tilde{g}_{1}$ and $\tilde{g}_{2}$, for $k$ in $D^{+}$, to obtain

$$
\begin{aligned}
i k \tilde{g}_{1}(\omega(k), t)=-k^{2} \tilde{g}_{0}(\omega(k), t) & +\frac{1}{\alpha(1-\alpha)}\left[\hat{q}_{0}\left(\alpha^{2} k\right)-\hat{q}_{0}(\alpha k)\right] \\
& +\frac{e^{\omega(k) t}}{\alpha(1-\alpha)}\left[\hat{q}(\alpha k, t)-\hat{q}\left(\alpha^{2} k, t\right)\right], \\
\tilde{g}_{2}(\omega(k), t)=-k^{2} \tilde{g}_{0}(\omega(k), t) & +\frac{1}{\alpha(1-\alpha)}\left[\alpha^{2} \hat{q}_{0}(\alpha k)-\alpha \hat{q}_{0}\left(\alpha^{2} k\right)\right] \\
& +\frac{e^{\omega(k) t}}{\alpha(1-\alpha)}\left[-\alpha^{2} \hat{q}(\alpha k, t)+\alpha \hat{q}\left(\alpha^{2} k, t\right)\right] .
\end{aligned}
$$

These expressions are substituted into the integral expression for $q(x, t)$, resulting in

$$
\begin{aligned}
q(x, t)= & \frac{1}{2 \pi} \int_{-\infty}^{\infty} e^{i k x-\omega(k) t} \hat{q}_{0}(k) d k-\frac{1}{2 \pi} \int_{\partial D^{+}} H(k, x, t) d k, \\
H(k, x, t)= & e^{i k x-\omega(k) t}\left[3 k^{2} \tilde{g}_{0}(\omega(k), t)-\alpha \hat{q}_{0}(\alpha k)-\alpha^{2} \hat{q}_{0}\left(\alpha^{2} k\right)\right] \\
& +e^{i k x}\left[-\alpha \hat{q}(\alpha k, t)-\alpha^{2} \hat{q}\left(\alpha^{2} k, t\right)\right] .
\end{aligned}
$$

We have used the fact that $\alpha$ is the cube root of unity and thus $\alpha^{3}=1$ and $1+\alpha+\alpha^{2}=0$.

5. Contribution of $\hat{q}(\nu(k), t)$. The functions $\hat{q}(\alpha k, t)$ and $\hat{q}\left(\alpha^{2} k, t\right)$ are bounded and analytic in $D^{+}$and decay to zero uniformly as $k \rightarrow \infty$. Once again Jordan's lemma implies that these terms do not contribute to the final solution, 
which is given by

$$
\begin{aligned}
q(x, t)= & \frac{1}{2 \pi} \int_{-\infty}^{\infty} e^{i k x-\omega(k) t} \hat{q}_{0}(k) d k-\frac{1}{2 \pi} \int_{\partial D^{+}} 3 k^{2} e^{i k x-\omega(k) t} \tilde{g}_{0}(\omega(k), t) d k \\
& +\frac{1}{2 \pi} \int_{\partial D^{+}} e^{i k x-\omega(k) t}\left[\alpha \hat{q}_{0}(\alpha k)+\alpha^{2} \hat{q}_{0}\left(\alpha^{2} k\right)\right] d k .
\end{aligned}
$$

The classical method of images approach, by which one obtains the sine transform solution to the heat equation, cannot be applied to this third-order PDE. Indeed it is not possible to obtain solutions via the method of images for any PDE which involves odd-order derivatives in $x$. For the third-order problem considered here, since $\hat{q}(\alpha k)$ is not bounded in the region $\{\arg (k) \in[0, \pi / 3]\}$, Jordan's lemma may not be applied in order to justify the deformation of the contour integral back to the real line. This apparently implies that an integral transform pair using integrals along the real line does not exist for this third-order PDE.

3.3. A (Slightly) Different Third-Order PDE. The two problems considered so far are well posed when only $q(0, t)$ is specified at the left boundary. Using a slight modification of the previous example we illustrate what changes when more boundary conditions are required. Consider the following third-order PDE on the positive halfline:

$$
q_{t}-q_{x x x}=0 .
$$

We assume that an initial condition $q(x, 0)$ has been given. The analysis of the global relation indicates the number and type of the boundary conditions that need to be prescribed in order for the problem to be well posed. The dispersion relation is

$$
\omega(k)=i k^{3},
$$

and

$$
D=\left\{k: \arg k \in\left(0, \frac{\pi}{3}\right) \cup\left(\frac{2 \pi}{3}, \pi\right) \cup\left(\frac{4 \pi}{3}, \frac{5 \pi}{3}\right)\right\} .
$$

Let $D^{+}=D_{1}^{+} \cup D_{2}^{+}$, where

$$
D_{1}^{+}=\left\{k: \arg k \in\left(\pi, \frac{\pi}{3}\right)\right\}, \quad D_{2}^{+}=\left\{k: \arg k \in\left(\frac{2 \pi}{3}, \pi\right)\right\},
$$

and

$$
D^{-}=\left\{k: \arg k \in\left(\frac{4 \pi}{3}, \frac{5 \pi}{3}\right)\right\}
$$

see Figure 3.5.

The local relation is given by

$$
\left(e^{-i k x+\omega(k) t} q\right)_{t}-\left(e^{-i k x+\omega(k) t}\left(q_{x x}+i k q_{x}-k^{2} q\right)\right)_{x}=0
$$

leading to the global relation (by integrating over the region $\mathcal{R}=\{x \geq 0,0<t \leq T\}$ and applying Green's theorem)

$$
\hat{q}_{0}(k)-\left[-k^{2} \tilde{g}_{0}(\omega(k), t)+i k \tilde{g}_{1}(\omega(k), t)+\tilde{g}_{2}(\omega(k), t)\right]=e^{\omega(k) t} \hat{q}(k, t), \quad \operatorname{Im}(k) \leq 0 .
$$




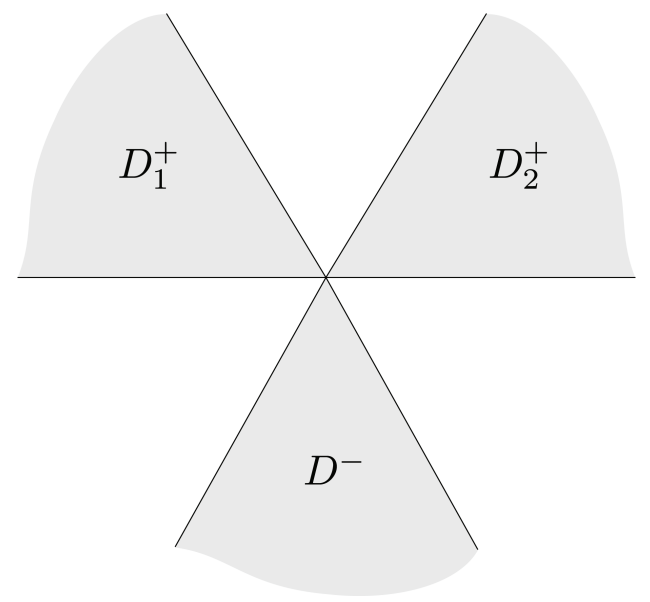

Fig. 3.5 Domain $D=\{k: \operatorname{Re}(\omega(k))<0\}$ for the third-order PDE (3.14).

The integral expression for the solution is

$$
\begin{aligned}
q(x, t)= & \frac{1}{2 \pi} \int_{-\infty}^{\infty} e^{i k x-\omega(k) t} \hat{q}_{0}(k) d k-\frac{1}{2 \pi} \int_{\partial D_{1}^{+}} e^{i k x-\omega(k) t} \tilde{g}(k, t) d k \\
& -\frac{1}{2 \pi} \int_{\partial D_{2}^{+}} e^{i k x-\omega(k) t} \tilde{g}(k, t) d k
\end{aligned}
$$

where

$$
\tilde{g}(k, t)=-k^{2} \tilde{g}_{0}(\omega(k), t)+i k \tilde{g}_{1}(\omega(k), t)+\tilde{g}_{2}(\omega(k), t) .
$$

The "deformation" from the real line to $\partial D^{+}$is obtained by adding to the path of integration the closed contour that is the boundary of the wedge between $D_{1}^{+}$and $D_{2}^{+}$ (see Figure 3.5) traversed in a clockwise sense. The contribution of the integrands along this contour is zero (Cauchy's theorem), and the contribution from the arc at infinity vanishes due to Jordan's lemma.

The symmetries of the global relation are as in the previous example. Hence if $k \in D_{1}^{+}, l \in D_{2}^{+}$, then $\alpha k, \alpha^{2} l \in D^{-}$, where $\alpha=e^{\frac{2 \pi i}{3}}$. We obtain the following versions of the global relation:

$$
\begin{array}{ll}
\hat{q}_{0}(\alpha k)+\alpha^{2} k^{2} \tilde{g}_{0}(\omega(k), t)-i \alpha k \tilde{g}_{1}(\omega(k), t)-\tilde{g}_{2}(\omega(k), t)=e^{\omega(k) t} \hat{q}(\alpha k, t), & k \in D_{1}^{+}, \\
(3.22) & \\
\hat{q}_{0}\left(\alpha^{2} k\right)+\alpha k^{2} \tilde{g}_{0}(\omega(k), t)-i \alpha^{2} k \tilde{g}_{1}(\omega(k), t)-\tilde{g}_{2}(\omega(k), t)=e^{\omega(k) t} \hat{q}\left(\alpha^{2} k, t\right), \quad k \in D_{2}^{+} .
\end{array}
$$

Hence in each region, $D_{1}^{+}$and $D_{2}^{+}$, there is one relation between the three quantities $\tilde{g}_{i}(\omega(k), t), i=0,1,2$. Thus two boundary conditions are required at the left boundary in order to be able to solve the global relation for the unspecified boundary condition. For instance, given $q(0, t)$ and $q_{x}(0, t)$ we can calculate $\tilde{g}_{0}(\omega(k))$ and $\tilde{g}_{1}(\omega(k))$. The above relations can be used to obtain two expressions for $\tilde{g}_{2}(\omega(k))$, one valid for $k \in D_{1}^{+}$and the other for $k \in D_{2}^{+}$. Substituting the resulting expressions into the integral expression for $q(x, t)$, we find the solution to (3.14) posed on the 
positive half-line. The difference in the number of boundary conditions required for the seemingly very similar boundary-value problems (3.10a) and (3.14) is explained as follows. For (3.10a), the phase velocity $-i \omega / k=-k^{2}$ is negative and the left boundary acts as a sink. Using (3.14), the phase velocity $-i \omega / k=k^{2}$ is positive, and the left boundary is a source. The same conclusion is reached using the group velocity, which is $3 \times$ the phase velocity.

3.4. A Multiterm Third-Order PDE. For the problems above, the symmetries of the dispersion relation are easily found. With this example we illustrate the use of Fokas's method if the symmetries are somewhat more complicated and the introduction of a branch cut is necessary. This method illustrates how to proceed in general, even if the symmetries cannot be written out explicitly. Consider

$$
q_{t}=q_{x}+q_{x x x}=0, \quad x>0,
$$

where an initial condition $q(x, 0)$ is given. The previous example indicates that two boundary conditions are required on the left boundary, which we will verify in the process of solving the equation. As in the previous example, we skip some calculation details that are similar to previous examples.

The dispersion relation is

$$
\omega(k)=-i k+i k^{3},
$$

and

$$
D=\left\{k: k_{I}\left(1+k_{I}^{2}-3 k_{R}^{2}\right)<0\right\},
$$

where $k_{R}$ and $k_{I}$ denote the real and imaginary parts of $k$, respectively. The region $D$ is shown on the left in Figure 3.6. The boundaries of the region consist of a hyperbola with asymptotes $k_{I}= \pm \sqrt{3} k_{R}$ and the real line. As above, we define $D^{+}\left(D^{-}\right)$to be the intersection of $D$ with the upper (lower) half-plane, and $D^{+}=D_{1}^{+} \cup D_{2}^{+}$, where

$$
D_{1}^{+}=\left\{k: D^{+} \cap \text { second quadrant }\right\}, \quad D_{2}^{+}=\left\{k: D^{+} \cap \text { first quadrant }\right\}
$$

as indicated in Figure 3.6.

The local relation is given by

$$
\left(e^{-i k x+\omega(k) t} q\right)_{t}-\left(e^{-i k x+\omega(k) t}\left(q_{x x}+i k q_{x}+\left(1-k^{2}\right) q\right)\right)_{x}=0
$$

integrating over the region $\mathcal{R}=\{x \geq 0,0<t \leq T\}$ and using Green's theorem gives the global relation

$$
\hat{q}_{0}(k)-\tilde{g}(k, t)=e^{\omega(k) t} \hat{q}(k, t), \quad \operatorname{Im}(k) \leq 0,
$$

where we have defined $\tilde{g}(k, t)=\left(1-k^{2}\right) \tilde{g}_{0}(\omega(k), t)+i k \tilde{g}_{1}(\omega(k), t)+\tilde{g}_{2}(\omega(k), t)$. At this point, we might write an integral expression for $q(x, t)$ involving a contour integral over the boundary of $D^{+}$, but first we examine the symmetries of the dispersion relation, as their functional form will influence what follows.

The discrete symmetries of $\omega(k)$ are found by solving $-i k+i k^{3}=-i \nu(k)+i \nu^{3}(k)$ for $\nu(k)$. Eliminating the solution $\nu(k)=k$, we find

$$
\nu=-\frac{k}{2} \pm \sqrt{1-\frac{3 k^{2}}{4}} .
$$



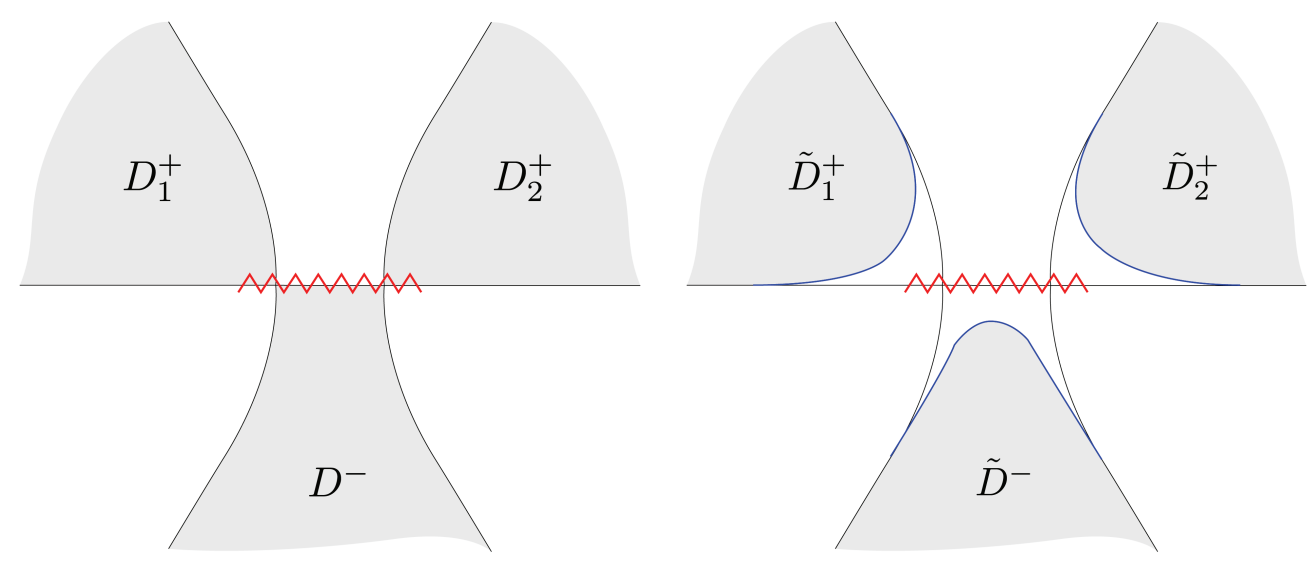

Fig. 3.6 Domain $D=\{k: \operatorname{Re}(\omega(k))<0\}$ (left) and its deformation $\tilde{D}$ (right) for the third-order $P D E$ (3.23). The branch cut $[-2 / \sqrt{3}, 2 / \sqrt{3}]$ is indicated by the jagged line.

This is a two-sheeted expression with branch points at $\pm \sqrt{3} / 2$, leading to a choice of branch cut along $[-\sqrt{3} / 2, \sqrt{3} / 2]$. Define $\nu_{1}$ to be the branch of $\nu$ which limits to $(-1 / 2+i \sqrt{3} / 2) k=k \exp (2 \pi i / 3)$, and let $\nu_{2}$ be the other branch, limiting to $k \exp (4 \pi i / 3)$. Our standard procedure would be to deform the integration over the real $k$ axis to one along $\partial D^{+}$, leading to the solution expression

$$
\begin{aligned}
q(x, t)= & \frac{1}{2 \pi} \int_{-\infty}^{\infty} e^{i k x-\omega(k) t} \hat{q}_{0}(k) d k-\frac{1}{2 \pi} \int_{\partial D_{1}^{+}} e^{i k x-\omega(k) t} \tilde{g}(k, t) d k \\
& -\frac{1}{2 \pi} \int_{\partial D_{2}^{+}} e^{i k x-\omega(k) t} \tilde{g}(k, t) d k
\end{aligned}
$$

where

$$
\tilde{g}(k, t)=\left(1-k^{2}\right) \tilde{g}_{0}(\omega(k), t)+i k \tilde{g}_{1}(\omega(k), t)+\tilde{g}_{2}(\omega(k), t) .
$$

This is problematic, since the integration paths $\partial D_{1}^{+}$and $\partial D_{2}^{-}$contain the branch points and part of the branch cut. Instead, we deform $D$ to $\tilde{D}$ with its constituent parts $\tilde{D}_{1}^{+}, \tilde{D}_{2}^{+}$, and $\tilde{D}^{-}$, so that the boundary of these domains is separated from the branch cut. Although the integrands are growing as $k \rightarrow \infty$ in $D$, they are analytic in any bounded region, and this deformation has no overall effect, due to Cauchy's theorem. The deformation from $D$ to $\tilde{D}$ can be chosen in many ways, as long as $\tilde{D}$ has the same asymptotic form as $D$. Specifically, we may deform $D_{1}^{+}$to $\tilde{D}_{1}^{+}$and induce deformations on $D_{2}^{+}$and $D^{-}$using $\tilde{D}_{2}^{+}=\nu_{2}\left(\tilde{D}_{1}^{+}\right)$and $D^{-}=\nu_{1}\left(\tilde{D}_{1}^{+}\right)$, respectively. This leads to the solution formula

$$
\begin{aligned}
q(x, t)= & \frac{1}{2 \pi} \int_{-\infty}^{\infty} e^{i k x-\omega(k) t} \hat{q}_{0}(k) d k-\frac{1}{2 \pi} \int_{\partial \tilde{D}_{1}^{+}} e^{i k x-\omega(k) t} \tilde{g}(k, t) d k \\
& -\frac{1}{2 \pi} \int_{\partial \tilde{D}_{2}^{+}} e^{i k x-\omega(k) t} \tilde{g}(k, t) d k .
\end{aligned}
$$


Next, we eliminate the dependence on unnecessary boundary conditions. In addition to (3.28), we have the following versions of the global relation:

$$
\begin{aligned}
& \hat{q}_{0}\left(\nu_{1}(k)\right)-\left(1-\nu_{1}(k)^{2}\right) \tilde{g}_{0}(\omega(k), t)-i \nu_{1}(k) \tilde{g}_{1}(\omega(k), t)-\tilde{g}_{2}(\omega(k), t)=e^{\omega(k) t} \hat{q}\left(\nu_{1}(k), t\right), \\
& \text { (3.31) } k \in \tilde{D}_{1}^{+} \text {, } \\
& \hat{q}_{0}\left(\nu_{2}(k)\right)-\left(1-\nu_{2}(k)^{2}\right) \tilde{g}_{0}(\omega(k), t)-i \nu_{2}(k) \tilde{g}_{1}(\omega(k), t)-\tilde{g}_{2}(\omega(k), t)=e^{\omega(k) t} \hat{q}\left(\nu_{2}(k), t\right), \\
& k \in \tilde{D}_{2}^{+} \text {. }
\end{aligned}
$$

As for the previous example, in each region, $\tilde{D}_{1}^{+}$and $\tilde{D}_{2}^{+}$, there is one relation among the three quantities $\tilde{g}_{i}(\omega(k), t), i=0,1,2$. Thus two boundary conditions are required at the left boundary in order to be able to solve the global relation for whichever boundary condition is unspecified.

3.5. A General Evolution PDE. Fokas's method is also applicable to the general constant coefficient linear evolution PDE

$$
q_{t}+\omega\left(-i \partial_{x}\right) q=0, \quad x \geq 0, t \in(0, T]
$$

Here $\omega(k)$ is a polynomial of degree $n$. To ensure that solutions do not grow in time, we impose that $\operatorname{Re}(\omega(k)) \geq 0$ for real $k$. Let

$$
\omega(k)=\alpha_{n} k^{n}+\alpha_{n-1} k^{n-1}+\cdots+\alpha_{0} .
$$

The large $k$ limit of the condition $\operatorname{Re}(\omega(k)) \geq 0, k \in \mathbb{R}$, implies that if $n$ is odd, then $\alpha_{n}= \pm i$. For $n$ even, $\operatorname{Re}\left(\alpha_{n}\right) \geq 0$. Using the dispersion relation we define the following regions in the complex $k$ plane:

$$
D=\{k: \operatorname{Re}(\omega(k))<0\}
$$

and

$$
D^{+}=D \cap \mathbb{C}^{+}, \quad D^{-}=D \cap \mathbb{C}^{-} .
$$

The local relation is given by

$$
\partial_{t}\left(e^{-i k x+\omega(k) t} q(x, t)\right)-\partial_{x}\left(e^{-i k x+\omega(k) t} \sum_{j=0}^{n-1} c_{j}(k) \partial_{x}^{j} q(x, t)\right)=0,
$$

where

$$
\sum_{j=0}^{n-1} c_{j}(k) \partial_{x}^{j} q(x, t)=\left.i\left(\frac{\omega(k)-\omega(l)}{k-l}\right)\right|_{l=-i \partial_{x}} q(x, t) .
$$

The proof is straightforward [9]. Equation (3.35) implies the global relation

$$
e^{\omega(k) T} \hat{q}(k, T)=\hat{q}_{0}(k)-\sum_{j=0}^{n-1} c_{j}(k) \tilde{g}_{j}(\omega(k), T), \quad \operatorname{Im}(k) \leq 0,
$$

where

$$
\tilde{g}_{j}(\omega, T)=\int_{0}^{T} e^{\omega s} \partial_{x}^{j} q(0, s) d s
$$


Applying the inverse Fourier transform to the global relation, we obtain the integral expression for the solution

$q(x, t)=\frac{1}{2 \pi} \int_{-\infty}^{\infty} e^{-i k x+\omega(k) t} \hat{q}_{0}(k) d k-\frac{1}{2 \pi} \int_{\partial D^{+}}\left(e^{-i k x+\omega(k) t} \sum_{j=0}^{n-1} c_{j}(k) \tilde{g}_{j}(\omega(k), t)\right) d k$.

In order to obtain a solution, we require expressions for the time transforms of the unknown boundary data valid for $k \in \partial D^{+}$. As with the previous examples, we use the discrete symmetries of the equation

$$
\omega(k)=\omega_{0} .
$$

This relation is a polynomial of order $n$ and thus has $n$ roots in the complex plane. The mappings from one root to another are precisely the transformations $k \rightarrow \nu(k)$ which leave $\omega(k)$ invariant. We employ the induced versions of the global relation to solve for the transforms of the unknown boundary data. The solution proceeds as before. Having eliminated the unknown boundary data, we obtain an expression that depends on $\hat{q}(\nu(k), t)$. The transformation $\nu$ is such that $\hat{q}(\nu(k), t)$ is analytic and bounded in the region $D^{+}$. Hence we may use the Cauchy integral theorem to eliminate the contribution of the term involving $\hat{q}(\nu(k), t)$ to the integral along $\partial D^{+}$.

It is possible to predict how many boundary conditions are required for a wellposed problem by considering the large $k$ behavior of $\omega(k)$. For large values of $k$, $\omega(k) \sim \alpha_{n} k^{n}$ and the region $D$ approaches

$$
D_{R}=\left\{k: \arg \alpha_{n}+n \arg k \in\left(\frac{\pi}{2}, \frac{3 \pi}{2}\right)+2 m \pi, \quad m=0, \ldots, n-1\right\} .
$$

Indeed for large $k, \operatorname{Re}(\omega(k)) \sim|k|^{n} \cos \left(\arg \alpha_{n}+n \arg k\right)$, which is negative for $k$ in $D_{R}$. The region $D_{R}$ consists of $n$ unbounded equal-angled sectors in the complex $k$ plane. Let $N$ represent the number of unbounded sectors of $D_{R}$ in the upper half-plane. It is easily seen that

$$
N= \begin{cases}n / 2, & n \text { even } \\ (n+1) / 2, & n \text { odd and } \alpha_{n}=-i \\ (n-1) / 2, & n \text { odd and } \alpha_{n}=i\end{cases}
$$

The reader is encouraged to verify the formulas for $D_{R}$ and $N$ for the examples presented above. Indeed Figures 3.2, 3.4, and 3.5 are examples of the three possible cases for $N$. In these examples, the regions $D_{R}$ and $D$ coincide.

In the integral expression for $q(x, t)$, the contour $\partial D^{+}$may be deformed to $\partial D_{R}^{+}=$ $\partial D_{R} \cap \mathbb{C}^{+}$, since the integrands are entire functions. Thus we require expressions for the time transform of the $n$ boundary data, valid in the unbounded sectors of $D_{R}$ in the upper half-plane.

Assume we are given $p$ boundary conditions at the boundary at $x=0$. This implies we require, for each of the $N$ sectors in $\mathbb{C}^{+}$, expressions for the transforms of the $n-p$ unknown boundary data. For each of the $N$ sectors in $\mathbb{C}^{+}$, the discrete symmetries of $\omega(k)$ allow us to choose $n-N$ transformations $\nu_{i}(k), i=0,1, \ldots, n-$ $N-1$, which map that sector to the $n-N$ remaining sectors. Hence, by substituting $\nu_{i}(k)$ for $k$, there are $n-N$ versions of the global relation valid in each of the $N$ 
sectors in $\mathbb{C}^{+}$. Thus we have $n-p$ unknowns with $n-N$ equations in each sector. It follows that we need $N$ boundary conditions at $x=0$ for a well-posed problem or, in other words, a well-posed problem on the positive half-line requires as many boundary conditions as there are sectors of $D_{R}$ in the upper half-plane.

Remarks.

- A canonical problem is one for which $q(0, t)$ and its first $N-1$ derivatives are provided as boundary conditions. An example of a noncanonical problem is the Neumann problem for the heat equation. Providing $N$ linear combinations with constant coefficients of a subset of the boundary values is another example (Robin problem). In this case, versions of the global relation valid in $D_{R}^{+}$and the $N$ linear combinations form a system of equations which can be solved for the boundary data, provided a certain determinant is not identically zero. If the determinant has zeros in $D_{R}^{+}$, then the contribution of these zeros to the final solution is computed via the residue theorem.

- For the class of the PDEs discussed in this paper, the dispersion relation $\omega(k)$ is a polynomial of degree $n$; see (3.34). Thus, by the fundamental theory of algebra, in addition to the identity, $n-1$ additional symmetries $\nu(k)$ exist, even if typically they cannot be written out in closed form. In this sense, the problem has been reduced to one of polynomial algebra.

\section{The Problem on the Finite Interval.}

4.I. The General Method. Having studied the problem posed on the half-line in some detail, we turn to the problem on the finite interval. We use the same notation as before, extending it where necessary to incorporate the boundary on the right-hand side:

$$
\begin{array}{rlrl}
q_{t}+\omega\left(-i \partial_{x}\right) q & =0, & (x, t) \in[0, L] \times[0, T], \\
q(x, 0) & =q_{0}(x), & x \in[0, L], \\
\partial_{x}^{j} q(0, t) & =g_{j}(t), & & j=0, \ldots, n-1, \\
\partial_{x}^{j} q(L, t) & =h_{j}(t), & & j=0, \ldots, n-1 .
\end{array}
$$

Here $g_{j}(t)$ and $h_{j}(t)$ denote the relevant boundary values at $x=0$ and $x=L$, respectively. We assume that $n$ of the functions $g_{j}(t)$ and $h_{j}(t)$ are given. In what follows, we will see that for a well-posed problem $N$ of these specified boundary functions should be specified at $x=0$ and the other $n-N$ at $x=L$, where $N$ is as in (3.37). As for the half-line problem, the different incarnations of the global relation (see below) obtained using the symmetries of the dispersion relation are used to eliminate unspecified boundary conditions.

As above, we define

$$
\tilde{g}_{j}(k, t)=\int_{0}^{t} e^{k s} g_{j}(s) d s, \quad \tilde{h}_{j}(k, t)=\int_{0}^{t} e^{k s} h_{j}(s) d s, \quad k \in \mathbb{C},
$$

where we have introduced the time transforms of both the left and right boundary values.

Employing the divergence form (3.35), we use Green's theorem on the domain $[0, L] \times[0, t]$. This is an easier domain to work with since it is bounded. The convergence of integrals over the spatial domain is not an issue, as it might be for problems posed on the half-line. We obtain the global relation

$$
\hat{q}_{0}(k)-\tilde{g}(k, t)+e^{-i k L} \tilde{h}(k, t)=e^{\omega(k) t} \int_{0}^{L} e^{-i k x} q(x, t) d x=e^{\omega(k) t} \hat{q}(k, t), \quad k \in \mathbb{C},
$$


with

$$
\tilde{h}(k, t)=\sum_{j=0}^{n-1} c_{j}(k) h_{j}(k, t), \quad \tilde{g}(k, t)=\sum_{j=0}^{n-1} c_{j}(k) g_{j}(k, t) .
$$

As before, the global relation is also valid if we replace $t$ by $T$. In contrast to the global relation for a problem on the half-line, the global relation (4.1) on the finite interval is valid for all $k \in \mathbb{C}$. It is clear this has important implications, as is discussed below.

To formally obtain a solution, we apply the inverse Fourier transform to (4.1):

$$
\begin{aligned}
q(x, t)= & \frac{1}{2 \pi} \int_{-\infty}^{\infty} e^{i k x} \hat{q}(k, t) d k \\
=\frac{1}{2 \pi} \int_{-\infty}^{\infty} e^{i k x-\omega(k) t} \hat{q}_{0}(k) d k & -\frac{1}{2 \pi} \int_{-\infty}^{\infty} e^{i k x-\omega(k) t} \tilde{g}(k, t) d k \\
& +\frac{1}{2 \pi} \int_{-\infty}^{\infty} e^{-i k(L-x)-\omega(k) t} \tilde{h}(k, t) d k .
\end{aligned}
$$

There are two major differences between the half-line problem and the problem on the finite interval. The most obvious is the addition of $\tilde{h}(k, t)$ due to the presence of the boundary on the right side of the domain. This provides extra algebraic complications in obtaining the solution. These complications are counteracted by the validity of the global relation in all of $\mathbb{C}$, which is the second major difference. This allows us to use $D^{+}$to eliminate the unknowns in $\tilde{h}(k, t)$, in addition to using $D^{-}$to eliminate the unknowns in $\tilde{g}(k, t)$ as we did earlier for the half-line. Proceeding with this intuition, we deform the contour of integration for the integral involving $\tilde{g}(k, t)$ up to $\partial D^{+}$as in the half-line case.

The third integral in (4.3) does not appear in the half-line case. The exponential $e^{-i k(L-x)}$ is bounded in the lower half-plane since $x \in[0, L]$. This observation allows us to analytically extend the integrand of the third term in (4.3) to the lower halfplane, at which point the path $(-\infty, \infty)$ can be deformed to the boundary of $D^{-}$using Jordan's lemma in $\mathbb{C}^{-} \backslash D^{-}$, in the same way that the deformation to the boundary of $D^{+}$was justified earlier in the half-line case. Taking our orientation convention into account we obtain

$$
\begin{aligned}
q(x, t)=\frac{1}{2 \pi} \int_{-\infty}^{\infty} e^{i k x-\omega(k) t} \hat{q}_{0}(k) d k & -\frac{1}{2 \pi} \int_{\partial D^{+}} e^{i k x-\omega(k) t} \tilde{g}(k, t) d k \\
& -\frac{1}{2 \pi} \int_{\partial D^{-}} e^{-i k(L-x)-\omega(k) t} \tilde{h}(k, t) d k .
\end{aligned}
$$

At this point, we have obtained the solution in terms of $2 n$ boundary values. We can prescribe only $n$ boundary conditions. The boundary values that are not given are eliminated using the symmetries of the dispersion relation as for the half-line case. As before, we have $n-1$ transformations that leave $\omega(k)$ invariant, i.e., there exist functions $\left\{\nu_{1}, \ldots, \nu_{n-1}\right\}$ such that $\omega(k)=\omega\left(\nu_{j}(k)\right)$. This provides $n$ equations with $2 n$ unknowns. If these equations may be solved for $n$ of the unknown boundary functions (or their $t$-transforms), then these functions may be eliminated in terms of the given boundary conditions. In [9] the conditions under which this system of equations can be solved in terms of a set of boundary functions to be eliminated is discussed. For instance, the system cannot be solved for the boundary functions on one boundary 
in terms of the boundary functions on the other boundary. Indeed, a well-posed problem on the finite interval requires boundary conditions at both boundaries. Of course, as for the half-line problem, using these different incarnations of the global relation introduces the quantities $\hat{q}\left(\nu_{j}(k), t\right), j=1, \ldots, n-1$, on the right-hand side of (4.4). This dependence is eliminated as before, using contour deformation and Jordan's lemma. These different steps are illustrated on a variety of examples below.

4.2. The Heat Equation with Homogeneous Dirichlet Boundary Conditions. Consider the problem

$$
\begin{array}{rlrl}
q_{t} & =q_{x x}, & x \in(0, L), & t \in(0, T], \\
q(x, 0) & =q_{0}(x), & x \geq 0, \\
q(0, t) & =0, & t \in[0, T], \\
q(L, t) & =0, & t \in[0, T] .
\end{array}
$$

As we have already seen, $\omega(k)=k^{2}$. Also, $\tilde{g}(k, t)=i k \tilde{g}_{0}\left(k^{2}, t\right)+\tilde{g}_{1}\left(k^{2}, t\right)=\tilde{g}_{1}\left(k^{2}, t\right)$ and $\tilde{h}(k, t)=i k \tilde{h}_{0}\left(k^{2}, t\right)+\tilde{h}_{1}\left(k^{2}, t\right)=\tilde{h}_{1}\left(k^{2}, t\right)$. The transformation that leaves $\omega(k)=k^{2}$ invariant is $k \rightarrow-k$; thus the two equations for the two unknowns $\tilde{g}_{1}\left(k^{2}, T\right)$ and $\tilde{h}_{1}\left(k^{2}, T\right)$ are

$$
\begin{aligned}
\hat{q}_{0}(k)-\tilde{g}_{1}\left(k^{2}, t\right)+e^{i k L} \tilde{h}_{1}\left(k^{2}, t\right) & =e^{k^{2} t} \hat{q}(k, t) \\
\text { and } \quad \hat{q}_{0}(-k)-\tilde{g}_{1}\left(k^{2}, t\right)+e^{-i k L} \tilde{h}_{1}\left(k^{2}, t\right) & =e^{k^{2} t} \hat{q}(-k, t) .
\end{aligned}
$$

These two equations are rewritten as

$$
\left[\begin{array}{cc}
-1 & e^{i k L} \\
-1 & e^{-i k L}
\end{array}\right]\left[\begin{array}{c}
\tilde{g}_{1}\left(k^{2}, t\right) \\
\tilde{h}_{1}\left(k^{2}, t\right)
\end{array}\right]=\left[\begin{array}{c}
e^{k^{2} t} \hat{q}(k, t)-\hat{q}_{0}(k) \\
e^{k^{2} t} \hat{q}(-k, t)-\hat{q}_{0}(-k)
\end{array}\right],
$$

from which

$$
\left[\begin{array}{c}
\tilde{g}_{1}\left(k^{2}, t\right) \\
\tilde{h}_{1}\left(k^{2}, t\right)
\end{array}\right]=\frac{1}{e^{i k L}-e^{-i k L}}\left[\begin{array}{cc}
e^{-i k L} & -e^{i k L} \\
1 & -1
\end{array}\right]\left[\begin{array}{c}
e^{k^{2} t} \hat{q}(k, t)-\hat{q}_{0}(k) \\
e^{k^{2} t} \hat{q}(-k, t)-\hat{q}_{0}(-k)
\end{array}\right] .
$$

Defining $\Delta(k)=e^{i k L}-e^{-i k L}$, we find

$\tilde{g}_{1}\left(k^{2}, t\right)=\frac{1}{\Delta(k)}\left[\hat{q}_{0}(-k) e^{-i k L}-\hat{q}_{0}(k) e^{i k L}\right]-\frac{1}{\Delta(k)}\left[e^{k^{2} t} \hat{q}(-k, t) e^{-i k L}-e^{k^{2} t} \hat{q}(k, t) e^{i k L}\right]$,

$\tilde{h}_{1}\left(k^{2}, t\right)=\frac{1}{\Delta(k)}\left[\hat{q}_{0}(-k)-\hat{q}_{0}(k)\right]-\frac{1}{\Delta(k)}\left[e^{k^{2} t} \hat{q}(-k, t)-e^{k^{2} t} \hat{q}(k, t)\right]$.

The final step is to justify neglecting the terms involving $\hat{q}( \pm k, t)$. For $\tilde{g}$, this is analogous to the case of the half-line, and it is not repeated here. However, we do demonstrate it for $\tilde{h}$. Consider the integral

$\int_{\partial D^{-}} e^{-i k(L-x)-k^{2} t} \frac{1}{\Delta(k)}\left[e^{k^{2} t} \hat{q}(-k, t)-e^{k^{2} t} \hat{q}(k, t)\right] d k=\int_{\partial D^{-}} e^{-i k(L-x)} \frac{1}{\Delta(k)}[\hat{q}(-k, t)-\hat{q}(k, t)] d k$,

where both $(\hat{q}(-k, t)-\hat{q}(k, t)) / \Delta(k)=\exp (-i k L)(\hat{q}(-k, t)-\hat{q}(k, t)) /(1-\exp (-2 i k L))$ and $\exp (-i k(L-x))$ decay to zero uniformly as $k \rightarrow \infty$ in the lower half-plane. Another application of Jordan's lemma shows that

$$
\int_{\partial D^{-}} e^{-i k(L-x)-k^{2} t} \frac{1}{\Delta(k)}\left[e^{k^{2} t} \hat{q}(-k, t)-e^{k^{2} t} \hat{q}(k, t)\right] d k=0,
$$




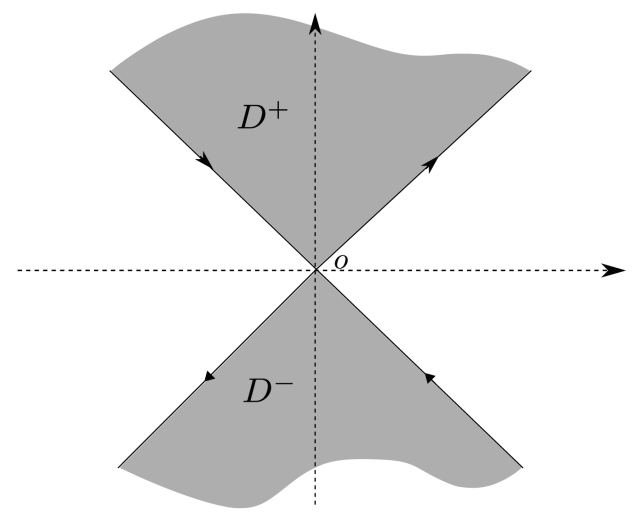

(a)

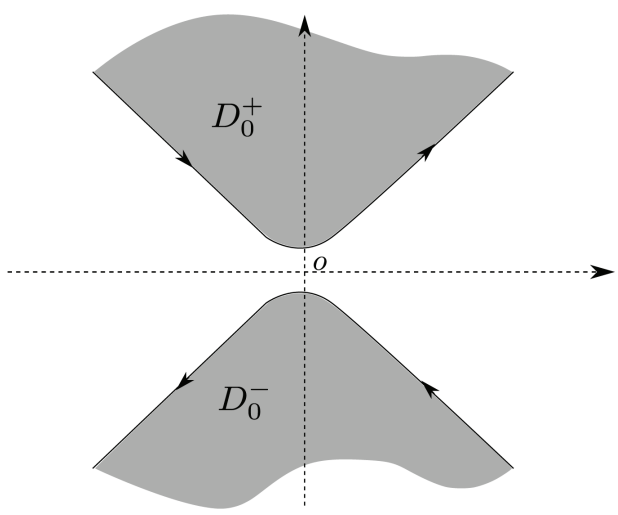

(b)

Fig. 4.I (a) The domains $D^{+}, D^{-}$and their respective boundaries. (b) The deformed domains $D_{0}^{+}$ and $D_{0}^{-}$, used to avoid the root of $\Delta(k)$ at the origin.

which implies

$$
\begin{aligned}
q(x, t) & =\frac{1}{2 \pi} \int_{-\infty}^{\infty} e^{i k x-k^{2} t} \hat{q}_{0}(k) d k \\
& -\frac{1}{2 \pi} \int_{\partial D^{+}} e^{i k x-k^{2} t} \frac{1}{\Delta(k)}\left[e^{i k L} \hat{q}_{0}(k)-e^{-i k L} \hat{q}_{0}(-k)\right] d k \\
& -\frac{1}{2 \pi} \int_{\partial D^{-}} e^{-i k(L-x)-k^{2} t} \frac{1}{\Delta(k)}\left[\hat{q}_{0}(k)-\hat{q}_{0}(-k)\right] d k .
\end{aligned}
$$

It should be noted that $\Delta(0)=0$ and $0 \in \partial D$, and thus it is convenient to deform the paths of integration, as shown in Figure 4.1. Having obtained the solution of (4.5) we demonstrate that it may be rewritten in the more familiar form of an infinite sum over a set of fundamental modes. It should be noted that this is not always possible. Indeed, for many problems a countable set of fundamental modes does not exist. The third-order equation of section 4.4 provides an example of this. Even in that case, the method of Fokas still provides a solution.

To reduce the integral representation (4.7) of the solution to an infinite series, we start by noticing that $\Delta(k)$ has simple zeros at a countable number of roots $k_{n}=\pi n / L$. The idea is to deform the paths of integration in (4.7) to a sequence of small loops around these roots, leading to a residue contribution for each loop, from which a series representation of the solution is obtained. In order for this to happen, all remaining integral contributions must vanish. For the heat equation, this is the case. In fact, this occurs for all problems where a series solution exists. For other problems (again, see section 4.4) the integral contribution to the solution does not vanish and little is gained by separating out a series part.

To ease the notation, define

$$
\begin{aligned}
& f^{+}(x, t, k)=\frac{1}{\Delta(k)}\left[e^{i k L} \hat{q}_{0}(-k)-e^{-i k L} \hat{q}_{0}(k)\right], \\
& f^{-}(x, t, k)=\frac{1}{\Delta(k)}\left[\hat{q}_{0}(-k)-\hat{q}_{0}(k)\right],
\end{aligned}
$$

where $f^{+}(x, k)$ and $f^{-}(x, k)$ appear in the integrands along $\partial D^{+}$and $\partial D^{-}$, respec- 
tively. We compute the residues at each $k_{n}$ for the integrand in the integral along $\partial D^{+}$. All poles are simple, and thus

$$
\begin{aligned}
c_{n}(x, t) & =\operatorname{Res}\left\{e^{i k x-k^{2} t} f^{+}(x, t, k), k=k_{n}\right\} \\
& =\frac{1}{\Delta^{\prime}\left(k_{n}\right)} e^{i k_{n} x-k_{n}^{2} t}\left[e^{i k_{n} L} \hat{q}_{0}\left(-k_{n}\right)-e^{-i k_{n} L} \hat{q}_{0}\left(k_{n}\right)\right] .
\end{aligned}
$$

Using $e^{i k_{n} L}=(-1)^{n}$ and $\Delta^{\prime}\left(k_{n}\right)=(-1)^{n} 2 i L$, we find

$$
c_{n}(x, t)=\frac{1}{2 i L} e^{i k_{n} x-k_{n}^{2} t}\left[\hat{q}_{0}\left(-k_{n}\right)-\hat{q}_{0}\left(k_{n}\right)\right] .
$$

This can be simplified using the explicit form of $\hat{q}_{0}(k)$. We have

$$
\hat{q}_{0}\left(k_{n}\right)-\hat{q}_{0}\left(-k_{n}\right)=-2 i \int_{0}^{L} \sin \left(k_{n} x\right) q_{0}(x) d x,
$$

which allows us to obtain a simple expression for the residue:

$$
c_{n}(x, t)=-\frac{1}{2} e^{i k_{n} x-k_{n}^{2} t} q_{n}, \quad q_{n}=\frac{2}{L} \int_{0}^{L} \sin \left(k_{n} x\right) q_{0}(x) d x .
$$

It remains to compute the residues of the integrand on $\partial D^{-}$. This is similar to the calculation just detailed, and we end up with residues equal to those already computed.

$$
\begin{aligned}
\operatorname{Res}\left\{e^{-i k(L-x)-\omega(k) t} f^{-}(x, t, k), k=k_{n}\right\} & =e^{i k_{n} x-\omega\left(k_{n}\right) t} \frac{1}{\Delta^{\prime}\left(k_{n}\right)}\left[\hat{q}_{0}\left(-k_{n}\right)-\hat{q}_{0}\left(k_{n}\right)\right] \\
& =\frac{1}{2 i L} e^{i k_{n} x-\omega\left(k_{n}\right) t}\left[-2 i \int_{0}^{L} \sin \left(k_{n} x\right) q_{0}(x) d x\right] \\
& =\operatorname{Res}\left\{e^{i k x-\omega(k) t} f^{+}(x, t, k), k=k_{n}\right\} .
\end{aligned}
$$

All the poles are on the real line. We deform the paths $\partial D^{ \pm}$back to the real line in order to collect their contributions. Additionally, in order to obtain a series solution, the first Fourier transform integral in (4.4) needs to be addressed. Using Jordan's lemma, we may deform back to the real line to end up with the contours in Figure 4.2. The solution can be written as

$$
\begin{aligned}
q(x, t) & =\frac{1}{2 \pi} \int_{-\infty}^{\infty} e^{i k x-k^{2} t} \hat{q}_{0}(k) d k \\
& -\frac{1}{2 \pi} \int_{\partial D_{\epsilon}^{+}} e^{i k x-k^{2} t} f^{+}(x, t, k) d k-\frac{1}{2 \pi} \int_{\partial D_{\epsilon}^{-}} e^{-i k(L-x)-k^{2} t} f^{-}(x, t, k) d k .
\end{aligned}
$$

Replacing the integrals with principal-value integrals and sums,

$$
\begin{aligned}
q(x, t) & =\frac{1}{2 \pi} \int_{-\infty}^{\infty} e^{i k x-k^{2} t} \hat{q}_{0}(k) d k \\
& -\left(\frac{1}{2 \pi} f_{-\infty}^{\infty} e^{i k x-k^{2} t} f^{+}(x, t, k) d k-\frac{\pi i}{2 \pi} \sum_{n=-\infty}^{\infty} \operatorname{Res}\left\{f^{+}(x, t, k), k=k_{n}\right\}\right) \\
& +\left(\frac{1}{2 \pi} f_{-\infty}^{\infty} e^{-i k(L-x)-k^{2} t} f^{-}(x, t, k) d k+\frac{\pi i}{2 \pi} \sum_{n=-\infty}^{\infty} \operatorname{Res}\left\{f^{-}(x, t, k), k=k_{n}\right\}\right) .
\end{aligned}
$$




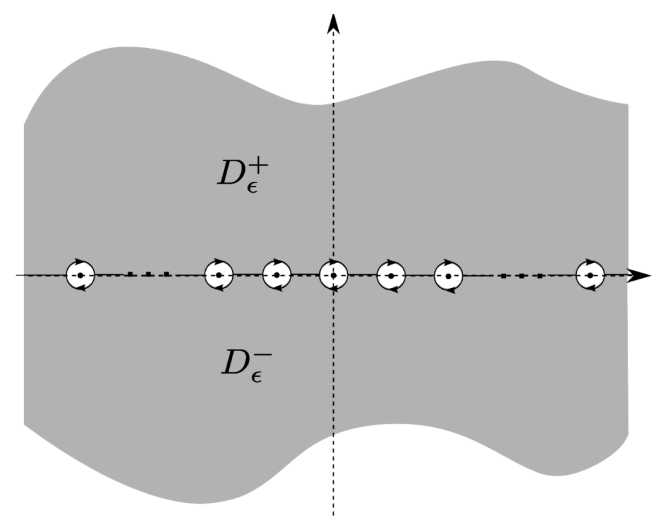

Fig. 4.2 Deformation to obtain Fourier series.

The two principal-value integrals sum to cancel the first integral, leaving us with

$$
q(x, t)=i \sum_{n=-\infty}^{\infty} c_{n}(x, t)=i \sum_{n=-\infty}^{\infty} \frac{1}{2} e^{i k_{n} x-k_{n}^{2} t}\left(-q_{n}\right) .
$$

Using $q_{n}=-q_{-n}$ and $q_{0}=0$, we find

$$
q(x, t)=\sum_{i=1}^{\infty} q_{n} e^{-k_{n}^{2} t} \sin \left(k_{n} x\right),
$$

which is the classical sine series solution.

4.3. The Heat Equation with Robin Boundary Conditions. Consider the somewhat more complicated problem

$$
\begin{array}{rlrl}
q_{t} & =q_{x x}, & (x, t) \in[0, L] & \times[0, T], \\
q(x, 0) & =q_{0}(x), & x & \in[0, L], \\
q_{x}(0, t)-\gamma q(0, t) & =0, & t & \in[0, T], \\
q_{x}(L, t)-\gamma q(L, t) & =0, & t & t \in[0, T],
\end{array}
$$

where $\gamma$ is real. The heat equation problem with Dirichlet or Neumann boundary data is straightforward using classical techniques and contains no surprises. The same is not true for the Robin problem, which contains an exponentially growing mode. We demonstrate how easily the method of Fokas extends to Robin boundary conditions, and how naturally the growing mode is captured. We work with homogeneous Robin conditions, to simplify the algebra involved. As before, $\omega(k)=k^{2}, \tilde{g}(k)=i k \tilde{g}_{0}\left(k^{2}\right)+$ $\tilde{g}_{1}\left(k^{2}\right)$, and $\tilde{h}(k)=i k \tilde{h}_{0}\left(k^{2}\right)+\tilde{h}_{1}\left(k^{2}\right)$. From the boundary conditions,

$$
\tilde{h}_{1}\left(k^{2}\right)=\gamma \tilde{h}_{0}\left(k^{2}\right), \quad \tilde{g}_{1}\left(k^{2}\right)=\gamma \tilde{g}_{0}\left(k^{2}\right) .
$$

Using $k \mapsto-k$ the two global relations are

$$
\begin{aligned}
\hat{q}_{0}(k)-\left[i k \tilde{g}_{0}\left(k^{2}\right)+\tilde{g}_{1}\left(k^{2}\right)\right]+e^{i k L}\left[i k \tilde{h}_{0}\left(k^{2}\right)+\tilde{h}_{1}\left(k^{2}\right)\right] & =e^{k^{2} t} \hat{q}(k, t), \\
\hat{q}_{0}(-k)-\left[-i k \tilde{g}_{0}\left(k^{2}\right)+\tilde{g}_{1}\left(k^{2}\right)\right]+e^{-i k L}\left[-i k \tilde{h}_{0}\left(k^{2}\right)+\tilde{h}_{1}\left(k^{2}\right)\right] & =e^{k^{2} t} \hat{q}(-k, t) .
\end{aligned}
$$


The equations (4.15)-(4.17) form a system of four linear equations for the four unknowns $\tilde{g}_{0}\left(k^{2}\right), \tilde{g}_{1}\left(k^{2}\right), \tilde{h}_{0}\left(k^{2}\right)$, and $\tilde{h}_{1}\left(k^{2}\right)$. These equations are valid in the whole complex $k$-plane, and all integrals involved are proper. Substitution of (4.15) in (4.16)-(4.17) gives

$$
\begin{aligned}
\tilde{g}_{0}\left(k^{2}\right)-e^{i k L} \tilde{h}_{0}\left(k^{2}\right) & =\frac{\hat{q}_{0}(k)-e^{k^{2} t} \hat{q}(k, t)}{\gamma+i k}, \\
\tilde{g}_{0}\left(k^{2}\right)-e^{-i k L} \tilde{h}_{0}\left(k^{2}\right) & =\frac{\hat{q}_{0}(-k)-e^{k^{2} t} \hat{q}(-k, t)}{\gamma-i k},
\end{aligned}
$$

so that

$$
\begin{aligned}
& g_{0}\left(k^{2}\right)=\frac{1}{\Delta(k)}\left[e^{i k L} \frac{\hat{q}_{0}(-k)-e^{k^{2} t} \hat{q}(-k, t)}{\gamma-i k}-e^{-i k L} \frac{\hat{q}_{0}(k)-e^{k^{2} t} \hat{q}(k, t)}{\gamma+i k}\right], \\
& h_{0}\left(k^{2}\right)=\frac{1}{\Delta(k)}\left[\frac{\hat{q}_{0}(-k)-e^{k^{2} t} \hat{q}(-k, t)}{\gamma-i k}-\frac{\hat{q}_{0}(k)-e^{k^{2} t} \hat{q}(k, t)}{\gamma+i k}\right],
\end{aligned}
$$

where, as before, $\Delta(k)=e^{i k L}-e^{-i k L}$. The solution formula (4.3) requires us to find $\tilde{g}$ and $\tilde{h}$ :

$$
\begin{aligned}
\tilde{g}(k) & =\frac{1}{\Delta(k)}\left[e^{i k L} \hat{q}_{0}(-k) \frac{\gamma+i k}{\gamma-i k}-e^{-i k L} \hat{q}_{0}(k)\right] \\
& -\frac{1}{\Delta(k)}\left[e^{i k L} e^{k^{2} t} \hat{q}(-k, t) \frac{\gamma+i k}{\gamma-i k}-e^{-i k L} e^{k^{2} t} \hat{q}(k, t)\right], \\
\tilde{h}(k) & =\frac{1}{\Delta(k)}\left[\hat{q}_{0}(-k) \frac{\gamma+i k}{\gamma-i k}-\hat{q}_{0}(k)\right]-\frac{1}{\Delta(k)}\left[e^{k^{2} t} \hat{q}(-k, t) \frac{\gamma+i k}{\gamma-i k}-e^{k^{2} t} \hat{q}(k, t)\right] .
\end{aligned}
$$

There is an added complication. Since $\gamma \in \mathbb{R}$ the individual terms in the above expressions have a pole in $D$ at $k=-i \gamma$, while $\tilde{g}(k)$ and $\tilde{h}(k)$ are analytic. Depending on the sign of $\gamma$ the pole at $k=-i \gamma$ is in $D^{+}$or in $D^{-}$. Either way it affects the use of Jordan's lemma, which is necessary to remove the terms involving $\hat{q}(k, t)$ and $\hat{q}(-k, t)$. Using the above expressions, the second and third integrals in (4.4) are split term-byterm. The resulting integrands are not analytic, and the contour deformation required to eliminate the contribution of $\hat{q}(-k, t)$ necessarily accounts for the pole singularity. For concreteness, let us assume that $\gamma<0$, thus $k=-i \gamma \in D^{+}$. We have two equivalent options. We can deform $\partial D^{+}$to pass above $k=-i \gamma$ or we can calculate a residue. To pursue this second option we use the fact that $e^{i k x-k^{2} t} \tilde{g}(k)$ is entire, so that

$$
\operatorname{Res}\left\{e^{i k x-k^{2} t} \tilde{g}(k), k=-i \gamma\right\}=0 .
$$

Therefore,

$$
\begin{aligned}
& \operatorname{Res}\left\{\frac{-1}{\Delta(k)}\left[e^{i k L} e^{k^{2} t} \hat{q}(-k, t) \frac{\gamma+i k}{\gamma-i k}-e^{-i k L} e^{k^{2} t} \hat{q}(k, t)\right], k=-i \gamma\right\} \\
= & \operatorname{Res}\left\{\frac{-1}{\Delta(k)}\left[e^{i k L} \hat{q}_{0}(-k) \frac{\gamma+i k}{\gamma-i k}-e^{-i k L} \hat{q}_{0}(k)\right], k=-i \gamma\right\}=\frac{-2 i \gamma}{\Delta(-i \gamma)} e^{\gamma L} \hat{q}_{0}(i \gamma) .
\end{aligned}
$$


The final expression for the solution $q(x, t)$ is

$$
\begin{aligned}
q(x, t)= & \frac{2 \gamma}{1-e^{-2 \gamma L}} \hat{q}_{0}(i \gamma) e^{\gamma x+\gamma^{2} t}+\frac{1}{2 \pi} \int_{-\infty}^{\infty} e^{i k x-k^{2} t} \hat{q}_{0}(k) d k \\
& -\frac{1}{2 \pi} \int_{\partial D_{0}^{+}} \frac{e^{i k x-k^{2} t}}{\Delta(k)}\left[e^{i k L} \hat{q}_{0}(-k) \frac{\gamma+i k}{\gamma-i k}-e^{-i k L} \hat{q}_{0}(k)\right] d k \\
& -\frac{1}{2 \pi} \int_{\partial D_{0}^{-}} \frac{e^{-i k(L-x)-k^{2} t}}{\Delta(k)}\left[\hat{q}_{0}(-k) \frac{\gamma+i k}{\gamma-i k}-\hat{q}_{0}(k)\right] d k,
\end{aligned}
$$

which displays the mode that is exponentially growing in time, which is explicitly present in this problem (see [15]).

Remarks.

- As in the case of the Dirichlet problem, we deform $\partial D^{+}$to $\partial D_{0}^{+}$to avoid the removable singularity at the origin.

- Using contour deformation and the residue theorem as in the previous example, the integrals in (4.18) may be evaluated explicitly, if so desired, resulting in a series solution (see equation (21) in section 4.3 of [15]).

4.4. A Third-Order Equation with Mixed Boundary Data. To demonstrate the true power of the Fokas method we consider a difficult problem whose explicit solution cannot be obtained using classical methods. Consider the problem

$$
\begin{aligned}
q_{t} & =q_{x x x}, & (x, t) \in[0, L] \times[0, T], \\
q(x, 0) & =q_{0}(x), & x \in[0, L], \\
q(0, t)-\alpha q_{x}(0, t) & =0, & t \in[0, T], \\
q(L, t)-\alpha q_{x}(L, t) & =0, & t \in[0, T], \\
q_{x x}(L, t) & =\beta, & t \in[0, T],
\end{aligned}
$$

where $\alpha$ and $\beta$ are real parameters. Classical methods [17] would proceed using separation of variables. This approach fails since a complete basis of discrete fundamental modes does not exist for this problem, as can be verified with some effort [9].

To find an explicit solution, we use the general solution (4.4), with $D$ as in Figure 4.3 , since $\omega(k)=i k^{3}$. Using the formulas (4.2) for $\tilde{g}$ and $\tilde{h}$,

$$
\begin{aligned}
\tilde{g}(\omega(k), t) & =(i k)^{2} \tilde{g}_{0}(\omega(k), t)+i k \tilde{g}_{1}(\omega(k), t)+\tilde{g}_{2}(\omega(k), t), \\
\tilde{h}(\omega(k), t) & =(i k)^{2} \tilde{h}_{0}(\omega(k), t)+i k \tilde{h}_{1}(\omega(k), t)+\tilde{h}_{2}(\omega(k), t) .
\end{aligned}
$$

The relations that leave $\omega(k)$ invariant are $k \mapsto \alpha k$ and $k \mapsto \alpha^{2} k$, where $\alpha=e^{2 i \pi / 3}$. We have introduced six boundary functions, $\tilde{h}_{j}$ and $\tilde{g}_{j}$, on which the solution $q(x, t)$ depends, according to (4.4). The BVP provides $h_{2}(k, t)=\beta \int_{0}^{t} e^{k s} d s$ and relations between $g_{0}(k, t)$ and $g_{1}(k, t)$, and between $h_{0}(k, t)$ and $h_{1}(k, t)$, respectively. It remains to find extra relations for the remaining boundary functions, using the invariances of the dispersion relation. As stated, the boundary function $h_{2}(k, t)$ is known. To determine the others we set up a system of five equations for five unknowns. Define $M(k, t)=\hat{q}_{0}(k)-e^{\omega(k) t} \hat{q}(k, t)-e^{i k L} \tilde{h}_{2}(\omega(k), t)$. Representing the system of equations 


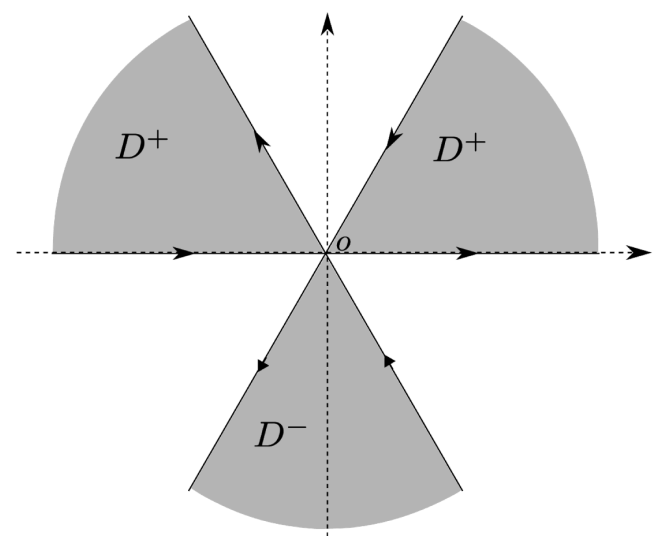

Fig. 4.3 $D$ for $q_{t}=q_{x x x}$.

in matrix form, we obtain

$$
\left[\begin{array}{ccccc}
k^{2} & -i k & -1 & -k^{2} e^{i k L} & i k e^{i k L} \\
\gamma^{2} k^{2} & -i \gamma k & -1 & -\gamma^{2} k^{2} e^{i \gamma k L} & i \gamma k e^{i \gamma k L} \\
\gamma k^{2} & -i \gamma^{2} k & -1 & -\gamma k^{2} e^{i \gamma^{2} k L} & i \gamma^{2} k e^{i \gamma^{2} k L} \\
1 & -\alpha & 0 & 0 & 0 \\
0 & 0 & 0 & 1 & -\alpha
\end{array}\right]\left[\begin{array}{c}
\tilde{g}_{0}(\omega(k), t) \\
\tilde{g}_{1}(\omega(k), t) \\
\tilde{g}_{2}(\omega(k), t) \\
\tilde{h}_{0}(\omega(k), t) \\
\tilde{h}_{1}(\omega(k), t)
\end{array}\right]=\left[\begin{array}{c}
-M(k, t) \\
-M(\gamma k, t) \\
-M\left(\gamma^{2} k, t\right) \\
0 \\
0
\end{array}\right]
$$

Once this system is solved, its solution is substituted into (4.4). At this point, the contributions from any poles are collected and Jordan's lemma is used to remove terms involving $\hat{q}(k, t)$, yielding the desired explicit solution. Although the algebra involved may be messy, the process is clear and is easily completed.

5. Conclusion. We have presented the application of Fokas's method to BVPs for linear evolution PDEs with constant coefficients on both the half-line and the finite interval. The main ideas behind the method are the use of (i) the analyticity properties of various functions, inherited from the global relation, and (ii) the discrete symmetries of the dispersion relation. We have illustrated that the method is more general than the standard methods used in the sense that it reproduces the results that they do, while being applicable to situations where the standard methods fail or are not applicable. We have illustrated that the method is suitable to be taught in the classroom at the undergraduate level.

Many other topics can be discussed at this point. Perhaps our biggest omission is not having mentioned Lax pairs. Indeed, it is possible to write a linear PDE with constant coefficients as the compatibility condition of two first-order ODEs for an auxiliary function $\psi(x, t)$ : one of these equations dictates how $\psi(x, t)$ changes as a function of $x$, with $t$ as a parameter, and the other equation has the roles of $x$ and $t$ reversed. Both equations may depend on $u(x, t)$ and its derivatives, and thus they are not autonomous. As shown in [9], this approach leads to the solution of the PDE via a scalar Riemann-Hilbert problem, which may be solved explicitly using the Plemelj formula [1]. Following this route, it is not necessary to introduce even the Fourier transform! Admittedly, the background in complex analysis required for this approach is far more extensive.

The method is far more general than we have discussed. For instance, it can be extended to apply to evolution equations with periodic boundary conditions [16], to 
evolution equations with more than one spatial dimension, or to elliptic equations. It is applicable also to some linear PDEs with nonconstant coefficients and, as already stated, to so-called integrable nonlinear equations. These topics are beyond the scope of this article, but the interested reader can find more details and additional references in [9]. The method continues to be extended. For instance, it was recently applied to elliptic PDEs in polar coordinates [14].

\section{REFERENCES}

[1] M. J. Ablowitz And A. S. Fokas, Complex Variables: Introduction and Applications, 2nd ed., Cambridge Texts Appl. Math., Cambridge University Press, Cambridge, UK, 2003.

[2] M. J. Ablowitz and H. Segur, Solitons and the Inverse Scattering Transform, SIAM Stud. Appl. Math. 4, SIAM, Philadelphia, PA., 1981.

[3] N. H. Asmar, Applied Complex Analysis with Partial Differential Equations, Prentice-Hall, Englewood Cliffs, NJ, 2002.

[4] S. J. FARLow, Partial Differential Equations for Scientists and Engineers, Dover, New York, 1993.

[5] N. Flyer AND A. S. Fokas, A hybrid analytical-numerical method for solving evolution partial differential equations. I. The half-line, Proc. R. Soc. Lond. Ser. A Math. Phys. Eng. Sci., 464 (2008), pp. 1823-1849.

[6] A. S. FoKAS, A unified transform method for solving linear and certain nonlinear PDEs, Proc. Roy. Soc. London Ser. A, 453 (1997), pp. 1411-1443.

[7] A. S. FoKAS, Integrable nonlinear evolution equations on the half-line, Comm. Math. Phys., 230 (2002), pp. 1-39.

[8] A. S. FoKAS, A new transform method for evolution partial differential equations, IMA J. Appl. Math., 67 (2002), pp. 559-590.

[9] A. S. Fokas, A Unified Approach to Boundary Value Problems, CBMS-NSF Regional Conf. Ser. in Appl. Math. 78, SIAM, Philadelphia, PA, 2008.

[10] A. S. Fokas And B. Pelloni, A transform method for linear evolution PDEs on a finite interval, IMA J. Appl. Math., 70 (2005), pp. 564-587.

[11] R. B. Guenther and J. W. Lee, Partial Differential Equations of Mathematical Physics and Integral Equations, Dover, Mineola, NY, 1996.

[12] R. Haberman, Elementary Applied Partial Differential Equations, Prentice-Hall, Englewood Cliffs, NJ, 1983.

[13] J. Kevorkian, Partial Differential Equations, The Wadsworth \& Brooks/Cole Mathematics Series, Wadsworth \& Brooks/Cole Advanced Books \& Software, Pacific Grove, CA, 1990.

[14] E. A. Spence And A. S. Fokas, A new transform method II: The global relation and boundaryvalue problems in polar coordinates, Proc. R. Soc. Lond. Ser. A Math. Phys. Eng. Sci., 466 (2010), pp. 2283-2307.

[15] W. A. Strauss, Partial Differential Equations, John Wiley \& Sons, Chichester, UK, 2008.

[16] T. Trogdon And B. Deconinck, The solution of linear constant-coefficient evolution PDEs with periodic boundary conditions, Appl. Anal., 91 (2011), pp. 529-544.

[17] H. F. Weinberger, A First Course in Partial Differential Equations with Complex Variables and Transform Methods, Dover, New York, 1995. 